\title{
Variación espacial y temporal de la diversidad y abundancia del fitoplancton del lago de Yojoa en un año hidrológico 2014-2015
}

\author{
Alba Isbela Hernández Oviedo, Mirna Marin ${ }^{1}$ \\ Lourdes Henriquez ${ }^{2}$ \\ Marcela Garay ${ }^{3}$
}

\section{RESUMEN}

El fitoplancton del lago de Yojoa estuvo representado por 81 especies: 59\% Cyanophyceae, 22\% Chlorophyceae, 9\% Bacillariophyceae, 4\% Zignematophyceae, $3 \%$ Euglenophyceae, 2\% Trebouxiophyceae y 1\% Dinophyceae.

La diversidad beta del fitoplancton del lago de Yojoa es relativamente alta independientemente del lugar y época de muestreo. La mayor riqueza específica correspondió a la estación frente al Hotel Brisas del Lago y la menor a Punto índice. Temporalmente, la mayor riqueza específica fue en Julio de 2014 y la menor en Enero de 2015, dominando: Microcystis aeruginosa, Aphanocapsa delicatissima, Aphanocapsa elachista, Oscillatoria limosa, Staurastrum leptocladum, Pseudoanabaena mucícola y Sphaerocystis schroeteri. Espacialmente, el mayor índice de dominancia correspondió a Quebrada Cianuro y el menor al Punto Índice. Temporalmente, el mayor índice de dominancia fue en Julio 2014 y el menor en época lluviosa y al final de la misma época. Espacialmente, la mayor similitud se encontró entre Puntilla y el Cacao y Punto Índice, y la menor entre la desembocadura del Río Varsovia y desembocadura Quebrada Cianuro. Temporalmente, la mayor similitud fue durante y a finales de época seca 2015, y la menor entre Julio 2014 y época seca 2015.

El promedio de la abundancia fue mayor en Mayo 2014 con 58,616 (Cel/ml), coincidiendo con el final de un evento de floración algal, sin embargo, disminuyó progresivamente durante el estudio. Al realizar el primer muestreo se observó una nata superficial color café verdoso correspondiente a algas filamentosas (Oscillatoria limosa y

1 Beneficiarias de una beca sustantiva de la DICYP. Profesoras de la Escuela de Biología, Facultad de Ciencias, UNAH: albaoviedo2001@yahoo.com, namirmarin@gmail.com

2 Beneficiaria de una beca sustantiva de la DICYP. Profesora del Instituto de Investigaciones en Microbiologia, Facultad de Ciencias, UNAH: Lourdes@unah.edu.hn

${ }^{3}$ Beneficiaria de una beca sustantiva de la DICYP. Profesora del Centro Universitario Regional del Litoral Atlántico (CURLA UNAH) marcelagarayc@gmail.com 
Lyngbya) en descomposición. La marcada estacionalidad del fitoplancton y las condiciones ambientales son los factores determinantes de las diferencias en la diversidad y abundancia encontrada en el lago de Yojoa.

Palabras clave: Fitoplancton variación, diversidad, abundancia, dominancia espacial, dominancia temporal fitoplancton, floración algal, lago de Yojoa.

\section{ABSTRACT}

The phytoplankton of Lake Yojoa was represented by 81 species: $59 \%$ Cyanophyceae, 22\% Chlorophyceae, 9\% Bacillariophyceae, 4\% Zignematophyceae, 3\% Euglenophyceae, $2 \%$ Trebouxiophyceae and $1 \%$ Dinophyceae.

The beta phytoplankton diversity of Lake Yojoa is relatively high regardless of sampling site and time. The highest specific wealth corresponded to the station in front of the Brisas del Lago Hotel and the lowest to index point. Temporally, the greatest specific richness was in July 2014 and the lowest in January 2015, dominating: Microcystis aeruginosa, Aphanocapsa delicatissima, Aphanocapsa elachista, Oscillatoria limosa, Staurastrum leptocladum, Pseudoanabaena mucícola and Sphaerocystis schroeteri. Spatially, the highest index of dominance corresponded to Quebrada Cianuro and the lowest to Index Point. Temporally, the highest index of dominance was in July 2014 and the lowest in September and November 2014. Spatially, the greatest similarity was found between Puntilla and Cacao and Point Index, and the lowest between the mouth of the Varsovia River and the Quebrada Cianuro mouth. Temporally, the greatest similarity was during and at the end of the dry season 2015, and the lowest between July 2014 and the dry season 2015.

The average abundance was higher in May 2014 with 58,616 (Cel / ml), coinciding with the end of an algal bloom event, however, declined progressively during the study. During the first sampling, a greenish-brown superficial cream corresponding to filamentous algae (Oscillatoria limosa and Lyngbya) was observed in decomposition. The marked seasonality of phytoplankton and environmental conditions are the determinants of differences in diversity and abundance found in Lake Yojoa.

Keywords: Phytoplankton variation, diversity, abundance, spatial dominance, temporal dominance phytoplankton, algal bloom, Yojoa lake. 


\section{INTRODUCCIÓN}

El primer eslabón en las cadenas tróficas de aguas continentales son los organismos fitoplanctónicos y son indicadores de su primer nivel productivo. La distribución de la mayoría de las algas dulceacuícolas es cosmopolita, sus características morfológicas y requerimientos nutritivos lo hacen el principal sintetizador de los mecanismos acuáticos (Hutchinson, 1967).

El fitoplancton está compuesto por grupos de algas en los sistemas acuáticos, algunas de las cuales son planctónicas y presentan locomoción por medio de flagelos y mecanismos hidrodinámicos que les permite distribuirse a lo largo y ancho de la columna de agua, sin embargo, la mayoría flota libremente en la zona litoral y pelágica de ecosistemas lénticos. Este grupo, junto a las macrófitas y algas adheridas algún sustrato (perifiton), (Wetzel, 1981 y Margalef, 1983) forman parte de los productores de estos ecosistemas y representan variaciones de acuerdo al grupo primario y sus requerimientos fisiológicos, manteniendo niveles de tolerancia a las condiciones ambientales fluctuantes (Wetzel, 1981, Gonzáles, 1988); lo cual contribuye, en parte, a la presencia, distribución, abundancia, diversidad y dominancia de las mismas.

Las respuestas de estos organismos a los cambios ambientales anuales son evidentes. En latitudes tropicales donde la luz y la temperatura son relativamente constantes en el curso del año, las variaciones estacionales dependen de la precipitación durante la época lluviosa y la época seca, ya que contribuye a los cambios hidrodinámicos del sistema, favoreciendo la circulación de las masas de agua y la generación de numerosas corrientes (Hernández y otros, 2008). Por otra parte, Vila et al., (1987), Margalef (1983), Salmaso (2000) y Prosperi (1999 y 2000), mencionan que en condiciones estables de los ecosistemas tropicales, las comunidades fitoplanctónicas tienden a alcanzar rangos de fluctuaciones bajas en su composición, abundancia, distribución y variación espacial y temporal.

Sin embargo, cualquier cambio en los parámetros ambientales que afectan el ecosistema acuático o que son parte de él, producen fluctuaciones en el comportamiento del fitoplancton. Las causas podrían ser la temperatura, cantidad y duración de la luz, $\mathrm{pH}$ y, sobre todo, el aporte de nutrientes al sistema, como fósforo, nitrógeno y sílice, procedentes de fuentes naturales y artificiales; de allí que un aumento descontrolado de las densidades poblacionales del fitoplancton puede provocar grandes problemas al equilibrio trófico del ecosistema. 
De acuerdo a De Lanza et al., (2000), el estudio del fitoplancton es importante en los lagos debido a que se utilizan como bioindicadores, ya que cuantifican la magnitud del estrés, de las características del hábitat y la respuesta ecológica al daño de un ecosistema. En ese sentido, se considera de gran importancia el conocimiento de la variación espacial y temporal de la diversidad y de la abundancia del fitoplancton del lago de Yojoa.

Las investigaciones biológicas del lago de Yojoa, aún las de carácter general, son escasas; abordan aspectos sobre todo de macroflora, peces (taxonomía y ecología) y contaminación del agua. Son aún menos las que incluyen particularidades de su fitoplancton y ninguna ha aplicado un enfoque multivariado para examinar la organización de las comunidades de algas con base en datos de presencia-ausencia de sus especies, variaciones estacionales, su relación a variables fisicoquímicas. Por esto, la determinación de la frecuencia relativa de la comunidad algal en estudios cualitativos durante las épocas del año es de gran importancia para presentar los cambios en la estructura de la comunidad y abundancia, así como conocer las condiciones ambientales que pueden dar lugar a la sucesión fitoplanctónica en ecosistemas acuáticos. Existe muy poca información sobre el fitoplancton del lago de Yojoa y a la fecha no se ha publicado ningún estudio sistemático sobre este tema.

En este trabajo se pretende aportar información nueva sobre la composición y variación espacial y temporal de la diversidad y abundancia de la comunidad fitoplanctónica en el lago de Yojoa. Esta información ayudaría a guiar la toma de decisiones sobre el manejo y conservación de este ecosistema de gran importancia para Honduras, especialmente para los habitantes que residen en la zona de influencia. Asimismo, aportará conocimiento científico y práctico sobre la temática y los hallazgos que se deriven del mismo servirán para ser aplicados en otros lagos o embalses de nuestro país.

El lago de Yojoa es el único lago natural y una de las reservas de agua dulce más importantes del país (House, 2002), cuya belleza alberga una gran diversidad de flora y fauna. La cuenca del lago de Yojoa contiene dos parques nacionales (Parque Nacional de Santa Bárbara con 132 km2 y el Parque Cerro Azul Meámbar con 209 km2), además esta subcuenca posee un valor ecológico y económico. Los bosques que rodean a la subcuenca del lago juegan un papel importante en el ciclo hidrológi$\mathrm{co}$, ya que la cantidad de precipitación ha dado lugar a la generación de hidroeléctricas (la represa de La Pita y la desviación de los ríos Yure y Varsovia, aumentaron la cuenca tributaria de Yojoa en aproximadamente un $27 \%$ ) en la zona, ejerciendo cambios drásticos en las propiedades hidrológicas del lago. Además, el lago de Yojoa 
fue declarado como la quinta zona protegida en 1971 y en el 2005 la convención de humedales RAMSAR declaró un área de 43,640 ha de la subcuenca como área protegida (Rivera, 2003). En el año 2007 se realizó la actualización de la batimetría (medición de profundidades) del lago con un volumen total de $1.40 \mathrm{~km} 3$; el área del espejo de agua en 8,475.80 ha, con profundidades de 22 hasta los $27.3 \mathrm{~m}$ (Romero y Mejía, 2007).

\section{MATERIALES Y MÉTODOS}

\section{Área de estudio}

La subcuenca del lago de Yojoa se define, en término estructural, como la parte meridional del 'graben' (término alemán que indica una cuenca estructural delimitada por fallas normales) del valle de Sula. Otro graben de dimensiones menores es el Mochito, que se encuentra posicionado al oeste del lago (Geólogos del mundo, 2011).

El lago de Yojoa se encuentra localizado a $650 \mathrm{msnm}$ entre los departamentos de Comayagua, Cortés y Santa Bárbara. Presenta las características de un lago monomíctico, con una longitud máxima de 15.6 kilómetros y una anchura máxima de 7.05 kilómetros, con una área de 81.33 kilómetros cuadrados, un perímetro de 57.56 kilómetros, una profundidad promedio de 16.57 metros, una profundidad máxima de 27 metros y un desarrollo litoral de 1.85 metros.

Actualmente, se han identificado 10 fuentes superficiales que aportan agua al lago de Yojoa (Canal Varsovia, quebrada Las Marías, quebrada El Cacao, quebrada Las Peñitas, quebrada Monteverde, canal Yure, río Blanco, quebrada de Balas, quebrada Raíces o quebrada Cianuro y quebrada de Jutiapa), una fuente superficial de desagüe, el canal Cañaveral que alimenta la represa Cañaveral, la que finalmente se une al río Lindo, un afluente del río Ulúa.

El lago de Yojoa es considerado como un lago cálido-monomíctico (mezcla sus aguas una vez al año), con enriquecimiento de nutrientes, principalmente nitratos y fosfatos, lo que resulta en un aumento de la producción primaria (especialmente proliferación de algas) y disminución en la concentración de oxígeno debido a la descomposición del material orgánico (Studer, 2007).

Se seleccionaron seis estaciones de muestreo, que coinciden con las estaciones de 
investigación y monitoreo que realiza periódicamente la Empresa Nacional de Energía Eléctrica (ENEE) en el lago de Yojoa (ver figura 1):

1. Estación 1 (E1). Desembocadura del río Varsovia (Lat.16P394001, Long. 1636748, Prof. $11.54 \mathrm{~m}$ ), frente a la desembocadura del canal Varsovia. El canal Varsovia y la quebrada Las Marías provienen de una zona muy poblada con mucha actividad agrícola.

2. Estación 2 (E2). Quebrada Jutiapa entre la Puntilla y el Cacao (Lat.16P393792, Long. 1641405, Prof. $14.6 \mathrm{~m}$ ), frente a la desembocadura de la quebrada de Jutiapa, está influenciada por los efluentes de las ventas de pescado frito en la margen este del lago de Yojoa.

3. Estación 3 (E3). Punto índice (Lat.16P394268, Long. 1645424, Prof. 15.4m), conocida también como estación índice, está influenciada por las desembocaduras del canal Yure y las quebradas Las Peñitas y Monte Verde; es una zona muy poblada con mucha actividad agrícola y ganadera, con varios comedores, restaurantes y hoteles. En la zona cercana a este punto de muestreo, la Empresa Aquafinca Saint Peter Fish, tiene instaladas aproximadamente 100 jaulas en balsas para el cultivo de peces.

4. Estación 4 (E4). Desembocadura de la quebrada Cianuro (Lat.16P389829, Long.1646073, Prof. 9.36 m), frente a la desembocadura de la quebrada Raíces, proveniente de la mina El Mochito; existe mucha actividad agrícola y ganadera en la microcuenca.

5. Estación 5 (E5). Desembocadura río Helado (Lat.16P383926, Long. 1650669, Prof. $12.2 \mathrm{~m}$ ), llamado también Las ventanas, localizada frente a la desembocadura de río Blanco con influencia de la quebrada de Balas; ambas quebradas provenientes de una zona agrícola y ganadera. Este punto de muestreo está localizado frente al inicio del drenaje del lago de Yojoa (canal Cañaveral).

6. Estación 6 (E6). Frente al Hotel Brisas del Lago (Lat.16P391890, Long. 1650614, Prof. $13.8 \mathrm{~m}$ ), a esta zona llegan los efluentes de las aguas residuales de hoteles, restaurantes y drenajes de lluvia e irrigación de la zona.

\section{Muestreo}

Se seleccionaron seis sitios de muestreo en todo el lago (ver figura 1). Para la ubicación de los sitios a muestrear, se tomó en cuenta el acceso, las características de la masa de agua para aspectos como la morfometría del lago, la profundidad, entradas de flujo, vegetación acuática y vertidos puntuales, tratando en lo posible de que los puntos a monitorear coincidieran con los sitios de la ENEE. 
La toma de las muestras para los análisis físicos-químicos se realizó en los mismos sitios en donde se tomaron muestras para determinar la comunidad fitoplanctónica. Estas se tomaron en campañas o muestreos bimensuales en un año hidrológico, iniciando a finales de la época seca de 2014 y finalizando a mediados de la época seca de 2015. Los muestreos fueron realizados en los sitios ubicados en la figura 1 y en las fechas siguientes:

1. El primer muestreo (M1): 31 de mayo de 2014 (al final de la época seca e inicios de la época lluviosa).

2. Segundo muestreo (M2): 12 de julio de 2014 (canícula).

3. Tercer muestreo (M3): 27 de septiembre de 2014 (época lluviosa).

4. Cuarto muestreo (M4): 01 de noviembre de 2014 (final de la época lluviosa e inicio de la época seca).

5. Quinto muestreo (M5): 24 de enero de 2015 (época seca).

6. Sexto muestreo (M6): 21 de marzo de 2015 (época seca).

En cada sitio se midieron variables fisicoquímicas como profundidad del agua, con un sonar Game Finder; temperatura del agua en oC y conductividad eléctrica a 20 oC $(\mu \mathrm{s} / \mathrm{cm} 2)$ con un conductímetro YSI desde la superficie hasta $10 \mathrm{~m}$. Fueron tomadas a diferentes profundidades $(0.5 \mathrm{~m})$, transparencia del disco de Secchi en $\mathrm{cm}$; oxígeno disuelto en (mg/l), con un oxinómetro YSI-DO200; porcentaje de saturación del oxígeno y se midió el pH en la superficie con un peachímetro Lamotte. Asimismo, se tomaron muestras de agua a $10 \mathrm{~cm}$ de la superficie con botellas Winkler para medir DBO5 (metodología standard).

Se tomaron muestras integradas de agua a la profundidad de la transparencia del disco de Secchi con la botella Van Dorn para determinar: la turbidez con el turbidímetro Mod. 2008-LaMotte, fósforo total (mg/l), fósforo soluble reactivo ( $\mathrm{mg} / \mathrm{l})$, ortofosfatos (mg/l), nitrógeno total $(\mathrm{mg} / \mathrm{l})$, dureza total $(\mathrm{mg} / \mathrm{l})$ y carbón orgánico total $(\mathrm{mg} / \mathrm{l})$. Las muestras fueron preservadas y procesadas en el laboratorio de hidrobiología de la Facultad de Ciencias, siguiendo los criterios HACH-EPA Compliant Monitoring Methods (40CFR 136.6).

Al momento del muestreo, se anotó el aspecto general de la masa de agua del lago, considerando la presencia de olor, color, aspecto y distribución de los organismos a simple vista; presencia de restos, costras, espumas y acumulaciones de algas. Las muestras para la determinación de nutrientes (fósforo total, fósforo soluble, ortofosfatos, nitrógeno total, dureza total y carbono orgánico total) fueron retiradas del refrigerador hasta alcanzar la temperatura ambiental. Seguidamente, se determinaron 
siguiendo la metodología especificada para cada nutriente en un espectrofotómetro modelo HACH DR3900. También se midió la turbidez con un turbidímetro LaMotte-2008 y se obtuvieron los valores de DBO5.

Para el análisis cualitativo del fitoplancton, las muestras fueron tomadas manualmente subsuperficialmente (30-50 cm de profundidad debajo de la superficie), con frascos plásticos de boca ancha de $250 \mathrm{ml}$ y mantenidas en una hielera hasta llegar al laboratorio en refrigeración. También se tomaron muestras con arrastre vertical desde la zona de transparencia del disco de Secchi hasta la superficie, con una red de plancton Wisconsin de $50 \mu \mathrm{m}$. De cada arrastre se tomaron 2 muestras en recipientes plásticos de $125 \mathrm{ml}$ con tapa de rosca, previamente rotulados con la información pertinente. Una de las muestras fue conservada en una hielera, en oscuridad para su posterior observación en vivo en un microscopio compuesto. La otra muestra se preservó con 14 gotas de solución de lugol acético y 5 gotas de formaldehído neutralizado al $4 \%$ para ser analizada posteriormente en el laboratorio.

Las muestras fueron extraídas del refrigerador hasta alcanzar la temperatura ambiental. Posteriormente se realizó la identificación de los organismos, para la cual se colocaron tres gotas de la muestra en un portaobjeto y posteriormente cubierta con un cubreobjetos. Se examinó toda el área con la ayuda de un microscopio compuesto Olympus y un micrométrico integrado, con aumentos de 10x, 40x y 100x; se identificaron todos los microorganismos siguiendo los criterios taxonómicos de la literatura especializada (Prescott, 1964; Ferguson, 1968; Whitford \& Schumacher, 1984; Edmonson, 1959; Prescott, 1962; APHA, AWWA \& WPCF, 1975; Bicudo \& Bicudo, 1970; Streble \& Krauter, 1985; Griffith, 1961; Anderson \& Weber, 1966; Riveray otros, 1982; Parra, Gonzales y Dellarosa,1983a; Parra, Gonzales y Dellarosa, 1983b; UNESCO, 2009; Sant'Anna, Célia Leite, et al. 2012).

Las muestras para el análisis cuantitativo del fitoplancton fueron tomadas con una botella muestreadora tipo Van Dorn de 1 litro; se tomaron tres muestras: una muestra hasta la profundidad de la transparencia del disco de Secchi, otra a la profundidad media y una última a $30 \mathrm{~cm}$ de la superficie. Se mezclaron en un contenedor plástico y se llenaron dos frascos limpios de $125 \mathrm{ml}$ con tapa de rosca. Una de las muestras se fijó con 14 gotas de lugol acético más 5 gotas de formaldehído neutralizado al 4 \%; la otra muestra se conservó en fresco y en la oscuridad. Ambas muestras fueron analizadas posteriormente en el laboratorio.

Las muestras refrigeradas y las preservadas con lugol acético fueron observadas e identificadas fueron observadas e identificadas utilizando tres gotas por muestra. 
Durante todo el estudio se analizaron un total de 180 muestras correspondientes a 6 muestras por sitio, durante seis muestreos realizados en un año hidrológico.

Figura 1. Mapa del lago de Yojoa con las estaciones de monitoreo, mayo de 2014-marzo de 2015

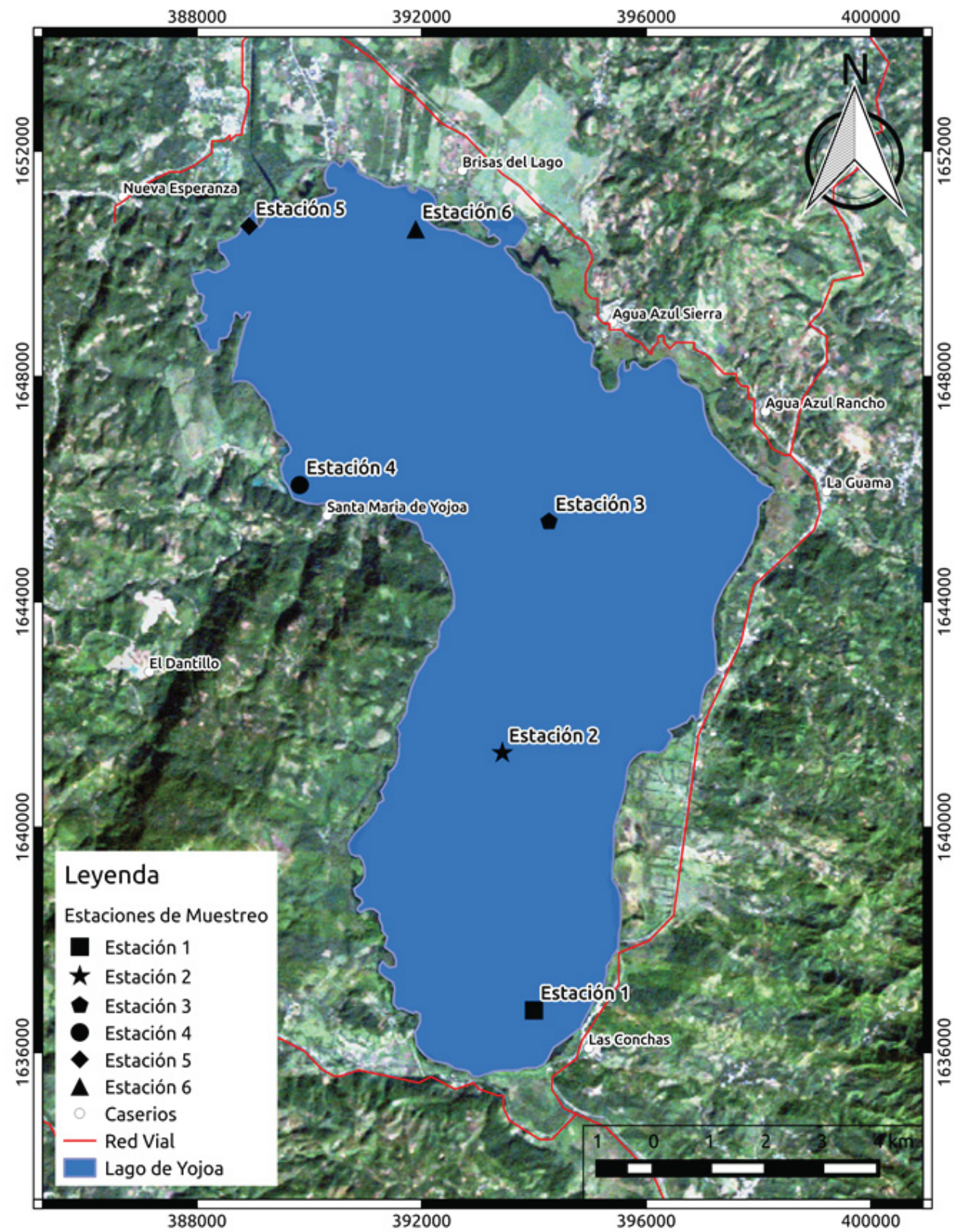

Fuente: Wiese Acosta, 2015 
Para el análisis cuantitativo y el recuento del fitoplancton se utilizó la cámara Sedgwick-Rafter y un microscopio compuesto Olympus con micrométrico integrado y con aumentos de 10x, 40x y 100x. El mecanismo de conteo consistió en extraer 10 $\mathrm{ml}$ de cada $125 \mathrm{ml}$ de muestra preservada, sedimentarla en tubos de vidrio por una hora. Posteriormente, se extrajo con una pipeta graduada una suspensión de $1 \mathrm{ml}$. Seguidamente, se llenó la cámara de Sedwick-Rafter, cubriéndola con un cubreobjetos y dejando reposar durante cinco minutos. Antes de iniciar el recuento con lente objetivo 10x, se observó detenidamente toda la extensión de la cámara para identificar las principales especies presentes en la muestra, tomando en cuenta que los organismos se dispersaran al azar a través del área de conteo.

Se contaron treinta (30) cuadrantes, ya que McAlice (1971) ha demostrado que en un recuento de 30 cuadrantes se puede determinar entre el 90 y el $95 \%$ de las especies presentes en una muestra. Las especies se cuantificaron por organismos, considerándose si era una célula, un filamento o una colonia (número de células/colonia), según el nivel de organización específica, midiendo cuidadosamente con el micrómetro el tamaño del organismo, colonia o filamento, tal como lo exige la clave taxonómica. Con el recuento se estimó la abundancia de organismos de cada especie previamente identificada.

Para calcular la población (T) entre las especies cuantificadas con la cámara de Sedwick-Rafter, se utilizó la fórmula $T=1000$. (C)/10. (N), en donde $\mathrm{C}$ es el número de organismos cuantificados en $\mathrm{N}$ (número de plazas), T se expresa como el número de organismos (o colonias) presentes en $1 \mathrm{ml}$ de la muestra, según el nivel de organización específico; también se tomó en cuenta el tamaño de la colonia. Posteriormente, se utilizó la metodología establecida (EPA, 2000) para realizar las conversiones respectivas en la variable $(C)$ de la fórmula indicada anteriormente. Los resultados espaciales y temporales del recuento de la comunidad fitoplanctónica se reportan en el Cuadro 6, ordenados por grupo taxonómico indicando la composición específica y la abundancia expresada en número de cel/ml contadas, factor que dependió de la cámara de conteo y del número de transectos contados por cámara.

Los datos estadísticos fueron analizados utilizando Excel y de acuerdo a la información de diferente literatura consultada. Se aplicaron varios índices para conocer la variación espacial y temporal de la estructura de la comunidad fitoplanctónica, tales como: índice de similitud de Jaccard, índice para riqueza de especie de Margalef y Whittaker (Berger \& Parker Dominance Index, 1970) y el análisis de frecuencia relativa y abundancia relativa (Southwood, 1978). Para calcular los índices anteriormente señalados se utilizaron datos cualitativos (abundancia de las especies más frecuen- 
tes) y cuantitativos (No. cel/ml) para obtener la abundancia. Asimismo, se correlacionaron variables abióticas y bióticas para saber el nivel de significancia, utilizando el Rs (coeficiente de Spearman) (Cramér, 1999) y para determinar la relación entre las especies y las estaciones del año (temporalidad) se aplicó el análisis de varianza (ANOVA) y test no paramétrico para las que no presentaron estos supuestos de distribución normal (García y López García, 1996); la hipótesis nula trabajada fue la independencia de la presencia-ausencia de las especies con respecto a las estaciones del año.

\section{RESULTADOS Y DISCUSIÓN}

El patrón de la precipitación pluvial en la subcuenca del lago de Yojoa es atípica, ya que no se ajusta a los patrones de las zonas vecinas. Es una de las zonas del país en donde se reportan las precipitaciones más altas; en el año 2014 se registraron precipitaciones anuales entre 2,303.88 y 3,353.5 mm. Los valores del promedio de la precipitación pluvial (en $\mathrm{mm}$ ) correspondientes a los meses en los que se realizaron los muestreos (Figura 2.) fueron calculados con los datos de precipitación provistos por la ENEE, correspondientes a las estaciones meteorológicas de Pito Solo, Santa Helena, El Jaral, El Planón, La Jutosa, El Cielito y Pedernales de la subcuenca del lago de Yojoa. Es posible observar las altas precipitaciones en mayo (1) de 2014, cuando teóricamente debían ser bajas.

Figura 2. Promedio de las precipitaciones del mes de los muestreos realizados en el lago de Yojoa 2014-2015

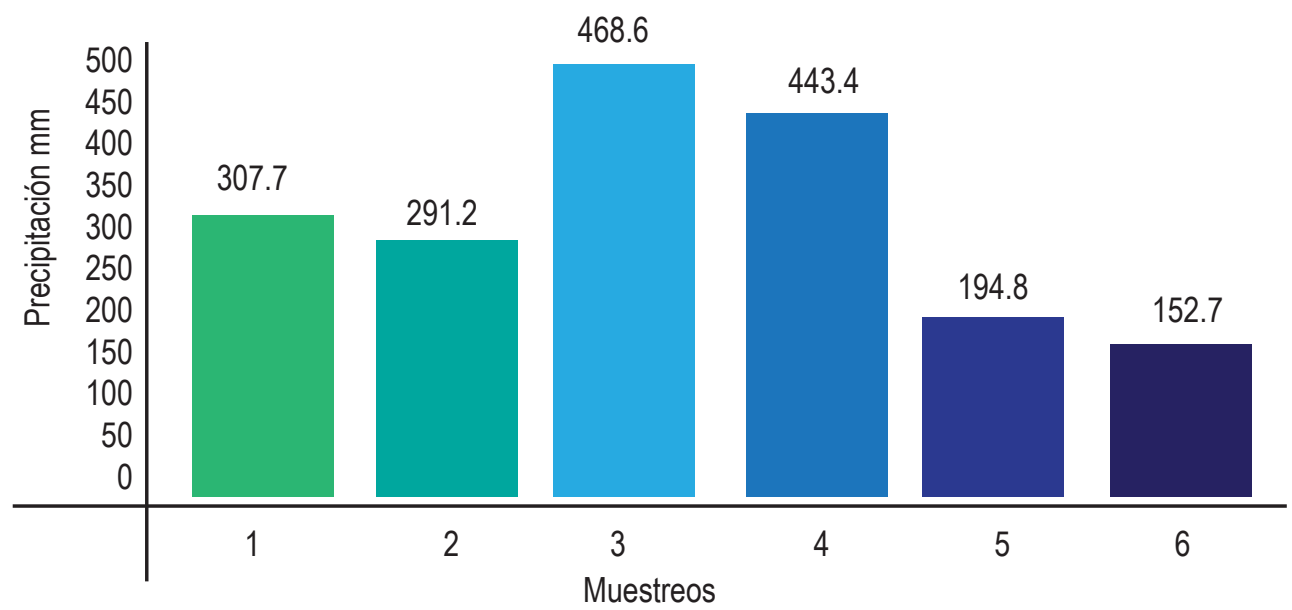


Cuadro 1. Parámetros ambientales del lago de Yojoa en los muestreos 2014-2015

\begin{tabular}{|c|c|c|c|c|c|c|c|c|c|c|c|c|}
\hline \multirow[b]{2}{*}{ MUESTREOS } & \multicolumn{6}{|c|}{ TEMPERATURA AMBIENTAL ( C) } & \multicolumn{6}{|c|}{ TEMPERATURA SUPERFICIAL DEL AGUA ( C ) } \\
\hline & M1 & M2 & M3 & M4 & M5 & M6 & M1 & $\mathrm{M} 2$ & M3 & M4 & M5 & M6 \\
\hline Promedio & 30.2 & 26.2 & 30.3 & 23.4 & 22.4 & 27.0 & 28.6 & 27.4 & 28.8 & 25.5 & 23.3 & 25.9 \\
\hline Max & 32,8 & 27,3 & 32,7 & 23,9 & 23,9 & 30,0 & 29.5 & 27.9 & 29.8 & 26.0 & 24.0 & 26.5 \\
\hline \multirow[t]{2}{*}{ Min } & 26,7 & 24,2 & 25,7 & 22,7 & 21,2 & 22,1 & 27.3 & 27.0 & 28.0 & 25.0 & 22.9 & 25.0 \\
\hline & \multicolumn{6}{|c|}{ HUMEDAD RELATIVA (\%) } & \multicolumn{6}{|c|}{ DISCO DE SECCHI (m) } \\
\hline MUESTREOS & M1 & M2 & M3 & M4 & M5 & M6 & M1 & $\mathrm{M} 2$ & $\mathrm{M} 3$ & M4 & M5 & M6 \\
\hline Promedio & 58.3 & 74.7 & 64.3 & 80.5 & 85.0 & 55.2 & 2.7 & 2.5 & 1.8 & 2.2 & 2.3 & 5.6 \\
\hline Max & 69 & 78 & 78 & 85 & 91 & 70 & 3,0 & 3,37 & 1,87 & 3,9 & 2,54 & 6,8 \\
\hline \multirow[t]{2}{*}{ Min } & 51 & 70 & 54 & 72 & 73 & 46 & 2,4 & 1,65 & 1,35 & 1,15 & 2,2 & 4,2 \\
\hline & \multicolumn{6}{|c|}{ TURBIDEZ ( NTU) } & \multicolumn{6}{|c|}{ CONDUCTIVIDAD $(\mu \Omega / \mathrm{cm})$} \\
\hline MUESTREOS & M1 & M2 & M3 & M4 & M5 & M6 & M1 & $\mathrm{M} 2$ & M3 & M4 & M5 & M6 \\
\hline Promedio & 4.5 & 1.0 & 4.2 & 7.4 & 3.3 & 1.7 & 86.3 & 118.6 & 112.5 & 101.1 & 92.3 & 125.9 \\
\hline Max & 9,4 & 2,1 & 9,5 & 18,3 & 4,2 & 2,8 & 115,0 & 129,0 & 119,0 & 110,0 & 100,0 & 130,0 \\
\hline \multirow[t]{2}{*}{ Min } & 3 & 0,4 & 2,4 & 2,9 & 2,4 & 1,0 & 11,0 & 100,0 & 110,0 & 90,0 & 80,0 & 120,0 \\
\hline & \multicolumn{6}{|l|}{$\mathrm{pH}$} & \multicolumn{6}{|c|}{ DUREZA TOTAL (mg/l) } \\
\hline MUESTREOS & M1 & $\mathrm{M} 2$ & $\mathrm{M} 3$ & M4 & M5 & M6 & M1 & $\mathrm{M} 2$ & M3 & M4 & M5 & M6 \\
\hline Promedio & 8.1 & 7.5 & 8.1 & 7.8 & 7.9 & 8.2 & 2.0 & 3.0 & 3.4 & 2.9 & 2.9 & 2.8 \\
\hline Max & 8,2 & 7,72 & 8,48 & 7,99 & 8,1 & 8,5 & 2,2 & 3,1 & 3,8 & 3,0 & 3,4 & 2,9 \\
\hline \multirow[t]{2}{*}{ Min } & 8,0 & 7,5 & 7,7 & 7,56 & 7,56 & 7,98 & 1,8 & 2,9 & 3,3 & 2,8 & 2,6 & 2,8 \\
\hline & \multicolumn{6}{|c|}{ FOSFORO REACTIVO (mg/l) } & \multicolumn{6}{|c|}{ FOSFORO TOTAL (mg/l) } \\
\hline MUESTREOS & M1 & $\mathrm{M} 2$ & $\mathrm{M} 3$ & M4 & M5 & M6 & M1 & $\mathrm{M} 2$ & M3 & M4 & M5 & M6 \\
\hline Promedio & 0.4 & 0.5 & 0.2 & 0.4 & 0.3 & 0.5 & 0.1 & 0.2 & 0.6 & 0.2 & 0.8 & 0.2 \\
\hline Max & 0,5 & 0,7 & 0,5 & 0,7 & 0,6 & 0,5 & 0,1 & 0,4 & 0,8 & 0,2 & 0,9 & 0,5 \\
\hline \multirow[t]{2}{*}{ Min } & 0,3 & 0,1 & 0,1 & 0,1 & 0,1 & 0,4 & 0,1 & 0,0 & 0,4 & 0,2 & 0,6 & 0,1 \\
\hline & \multicolumn{6}{|c|}{ NITROGENO TOTAL (mg/l) } & \multicolumn{6}{|c|}{ TOC (mg/l) } \\
\hline MUESTREOS & M1 & M2 & M3 & M4 & M5 & M6 & M1 & $\mathrm{M} 2$ & M3 & M4 & M5 & M6 \\
\hline Promedio & 6.6 & 0.7 & 0.4 & 0.6 & 2.7 & 2.2 & 8.4 & 5.2 & 3.2 & 19.3 & 3.8 & 4.3 \\
\hline Max & 34,7 & 2,1 & 1,1 & 0,9 & 3,9 & 3,9 & 20,0 & 11,5 & 4,6 & 19,9 & 7,2 & 14,3 \\
\hline \multirow[t]{2}{*}{ Min } & 0,0 & 0,0 & 0,0 & 0,0 & 1,5 & 0,6 & 1,6 & 2,3 & 2,2 & 18,9 & 0,0 & 1,9 \\
\hline & \multicolumn{6}{|c|}{ OXIGENO DISUELTO (ppm) } & \multicolumn{6}{|c|}{ DBO (mg/l) } \\
\hline MUESTREOS & M1 & M2 & M3 & M4 & M5 & M6 & M1 & $\mathrm{M} 2$ & M3 & M4 & M5 & M6 \\
\hline Promedio & 5.07 & 5.00 & 5.30 & 4.06 & 5.34 & 4.74 & 0.83 & 1.11 & 3.70 & 2.14 & 3.71 & 2.84 \\
\hline Max & 6,0 & 5,45 & 8,5 & 5,8 & 5,52 & 5,06 & 1,6 & 1,26 & 4,7 & 3,6 & 4,7 & 3,55 \\
\hline Min & 4,4 & 4,23 & 4,14 & 1,84 & 4,97 & 4,46 & 0,2 & 0,76 & 2,12 & 1,01 & 1,72 & 2,35 \\
\hline
\end{tabular}

Fuente propia

Los valores de los promedios de la temperatura ambiental en el periodo de estudio oscilaron entre los 22.4 oC en época seca (M5) y $30.2^{\circ} \mathrm{C}$ al final de la época seca e inicios de la época lluviosa (M1). El promedio de la temperatura superficial del agua varió entre $23.3^{\circ} \mathrm{C}$ al inicio de la época seca (M5) y $28.8^{\circ} \mathrm{C}$ en época lluviosa (M3).

Los promedios de la humedad relativa variaron entre $55.2 \%$ en época seca (M6) y $85.0 \%$ a finales de la época lluviosa e inicios de la época seca (M5). El promedio general de la transparencia del agua para el periodo de estudio fue de 2.9 metros. Los promedios de los valores de la profundidad del disco de Secchi durante el estudio oscilaron entre 1.8 metros en época lluviosa (M3) y 5.6 metros en época seca (M6). 
La gran diferencia de los datos observados en el último muestreo (5.6 metros) se explican por el aumento de las descargas para generación de energía relacionada con la precipitación alta en la subcuenca.

El promedio general de la conductividad fue de $107.8 \mu \Omega / \mathrm{cm}$. El promedio por muestreo varió entre $86.3 \mu \Omega / \mathrm{cm}$ a finales de época seca e inicios de época lluviosa (M1) y $125.9 \mu \Omega / \mathrm{cm}$. en época seca (M6). El promedio general de la turbidez fue de 4.0 NTU. El promedio por muestreo varió entre 1,0 NTU en época lluviosa (M2) y 7,4 NTU en época lluviosa (M4) 2015. El promedio de la profundidad de los sitios, medida en metros, varió entre 11 y 13 para el periodo de estudio, excepto M5 que registró $16 \mathrm{~m}$ y $\mathrm{M} 6$ con $15 \mathrm{~m}$.

El promedio general del oxígeno disuelto en el periodo de estudio fue de $4.92 \mathrm{ppm}$ y el promedio más alto se registró al final de la época lluviosa e inicios de la época seca (M5) con 5.34 ppm; el promedio más bajo correspondió al final de época lluviosa (M4) con $4.06 \mathrm{ppm}$. El promedio general de la demanda biológica oxígeno en el período de estudio fue de $2.4 \mathrm{mg} / \mathrm{l}$. El promedio más alto se registró al final de la época lluviosa e inicios de la época seca (M5) con 3,71 mg/l y el promedio más bajo $(0,83 \mathrm{mg} / \mathrm{l}) \mathrm{se}$ registró al final de la época seca e inicios de la estación lluviosa (M1).

Los valores promedios generales del $\mathrm{pH}$ en el periodo de estudio fueron ligeramente básicos (7.93). Los valores promedios variaron entre 7.5 en época lluviosa (M2) y 8.2 en época seca (M6). El promedio general de dureza total del lago de Yojoa en el periodo de estudio fue de $2.83 \mathrm{mg} / \mathrm{l}$. Los promedios de los valores de dureza total ( $\mathrm{mg} / \mathrm{l}$ ) variaron entre 2,0 al final de la época seca e inicios de la época lluviosa (M1) y 3.4 en la época lluviosa (M3).

El promedio general de concentraciones de fósforo reactivo en el periodo del estudio fue de $0,4 \mathrm{mg} / \mathrm{l}(400 \mathrm{mg} / \mathrm{m} 3)$. El promedio más alto $(0.5 \mathrm{mg} / \mathrm{l})$ fue durante la canícula (M2) y en la época seca (M6). El promedio más bajo de $0.2 \mathrm{mg} / \mathrm{l}$ se registró en época lluviosa (M3). El promedio general de concentraciones de fósforo total en el periodo de estudio fue de $0.3 \mathrm{mg} / \mathrm{l}(300 \mathrm{mg} / \mathrm{m} 3)$. El promedio más alto fue $0.8 \mathrm{mg} / \mathrm{l}$ en M5 a finales de la época lluviosa e inicios de la época seca y el promedio más bajo fue de $0.1 \mathrm{mg} / \mathrm{l}$ en M1 correspondiente al final de la época seca e inicio de la época lluviosa.

El promedio general de concentraciones de nitrógeno total en el periodo de estudio fue de $2.2 \mathrm{mg} / \mathrm{l}$. El promedio más alto fue $6.6 \mathrm{mg} / \mathrm{l}$ al final de la época seca e inicios de la época lluviosa (M1) y el más bajo de $0.4 \mathrm{mg} / \mathrm{l}$ en la época lluviosa (M3). El promedio general de concentraciones de carbono orgánico total para el periodo de 
estudio fue de $7.4 \mathrm{mg} / \mathrm{l}$. El promedio más alto de carbono orgánico total fue de 19.3 $\mathrm{mg} / \mathrm{l}$ y se registró al final de la época lluviosa e inicio de la época seca (M4) y el más bajo fue de 3,2 mg/l en la época lluviosa (M3).

\section{Cuadro 2. Composición taxonómica del fitoplancton en el lago de Yojoa, mayo de 2104 a marzo de 2015.}

\begin{tabular}{|c|c|c|c|}
\hline Clase & Orden & Familia & Género \\
\hline \multirow[t]{4}{*}{ Cyanophyceae } & Nostocales & Nostocaceae & $\begin{array}{l}\text { Dolichospermum solitarium (Klebahn) Wacklin, } \\
\text { L.Hoffmann \& Komárek } 2009 \\
\text { Dolichospermum spiroides (Klebhan) Wacklin, } \\
\text { L.Hoffmann \& Komárek } 2009 \\
\text { Dolichospermum circinalis (Rabenhorst ex Bornet } \\
\text { \& Flahault) P.Wacklin, L.Hoffmann \& J.Komárek } \\
2009 \\
\text { Dolichospermum sp. (Ralfs ex Bornet \& Flahault) } \\
\text { P.Wacklin, L.Hoffmann \& J.Komárek, } 2009\end{array}$ \\
\hline & \multirow[t]{3}{*}{ Chrooccocales } & Nostocaceae & $\begin{array}{l}\text { Microcystis aeruginosa (Kützing) Kützing } 1846 \\
\text { Microcystis flosaquae (Wittrock) Kirchner } 1898 \\
\text { Microcystis botrys Teiling } 1942 \\
\text { Microcystis potocystis W.B.Crow } 1923 \\
\text { Microcystis spp. Kützing, } 1833 \\
\text { Gleocapsa sp. Kützing, } 1843\end{array}$ \\
\hline & & Chroococcaceae & $\begin{array}{l}\text { Synechocystis sp. C. Sauvageau, } 1892 \\
\text { Asterocapsa submersa Azevedo et al. } 2003 \\
\text { Chroococcus spp. Nägeli, } 1849 \\
\text { Chroococcus limneticus Lemmermann } 1898 \\
\text { Chroococcus dispersus Lemmermann } 1904\end{array}$ \\
\hline & & Synechoccaceae & $\begin{array}{l}\text { Aphanocapsa endophytica G.M.Smith } 1920 \\
\text { Aphanocapsa delicatissima West \& G.S.West } \\
1912 \\
\text { Aphanocapsa mucicola (Meneghini) Wille } 1919 \\
\text { Aphanocapsa holsatica (Lemmermann) G.Cron- } \\
\text { berg \& Komárek } 1994 \\
\text { Aphanocapsa koordesii Strom, } 1923 \\
\text { Aphanocapsa planctonica (G.M.Smith) Komárek \& } \\
\text { Anagnostidis } 1995 \\
\text { Aphanocapsa incerta (Lemmermann) G.Cronberg } \\
\text { \& Komárek } 1994 \\
\text { Aphanocapsa elachista West \& G.S.West } 1894 \\
\text { Aphanocapsa spp. Nägeli, } 1849 \\
\text { Aphanothece spp. Nägeli, } 1849 \\
\text { Synechococcus nidulans (Pringsheim) Komárek in } \\
\text { Bourrelly } 1970 \\
\text { Gloeothece sp. Bornet } 1892\end{array}$ \\
\hline
\end{tabular}




\begin{tabular}{|c|c|c|c|}
\hline Clase & Orden & Familia & Género \\
\hline \multirow[t]{5}{*}{ Cyanophyceae } & \multirow[t]{2}{*}{ Oscillatoriales } & Oscillatoriaceae & $\begin{array}{l}\text { Oscillatoria limosa C.Agardh ex Gomont } 1892 \\
\text { Oscillatoria nigriviridis Thwaites ex Gomont } 1892 \\
\text { Oscillatoria prínceps Vaucher ex Gomont } 1892 \\
\text { Oscillatoria ornata Kützing ex Gomont } 1892 \\
\text { Oscillatoria germinata Schwabe ex Gomont } 1892 \\
\text { Oscillatoria sancta Kützing ex Gomont } 1892 \\
\text { Oscillatoria sp. Vaucher ex Gomont, } 1892 \\
\text { Lyngbya spirulinoidea Agardh ex Gomont, } 1892 \\
\text { Lyngbya virgey C.Agardh ex Gomont, } 1892 \\
\text { Lyngbya sp. C.Agardh ex Gomont, } 1892\end{array}$ \\
\hline & & Phormidiaceae & $\begin{array}{l}\text { Phormidium sp. Kützing ex Gomont, } 1892 \\
\text { Trichodesmium lacustre Klebahn } 1895 \\
\text { Planktothrix sp. K.Anagnostidis \& J.Komárek, } \\
1988\end{array}$ \\
\hline & \multirow[t]{3}{*}{ Synechococales } & Borziaceae & Borzia trilocularis Cohn ex Gomont 1892 \\
\hline & & Pseudanabaenaceae & $\begin{array}{l}\text { Leptolynghbya perelegans (Lemmermann) Ana- } \\
\text { gnostidis \& Komárek } 1988 \\
\text { Limnotrix sp. Meffert, } 1988 \\
\text { Pseudanabaena mucicola (Naumann \& Huber- } \\
\text { Pestalozzi) Schwabe } 1964 \\
\text { Pseudanabaena galeata Böcher } 1949 \\
\text { Pseudanabaena sp. Lauterborn, } 1915\end{array}$ \\
\hline & & Merismopediacea & $\begin{array}{l}\text { Coelosphaerium sp. Nägeli, } 1849 \\
\text { Limnococcus sp. Komárková et al. (2010) }\end{array}$ \\
\hline \multirow[t]{3}{*}{ Zygnemophyceae } & \multirow[t]{3}{*}{ Desmidiales } & Desmidiaceae & Staurastrum leptocladum Nordstedt 1870 \\
\hline & & Gloeobotrydiaceae & Cosmarium minutis Corda ex Ralfs, 1848 \\
\hline & & Tribonemataceae & Tribonema sp. Derbès \& Solier, 1851 \\
\hline \multirow[t]{5}{*}{ Chlorophyceae } & \multirow[t]{5}{*}{ Chlamydomonadales } & Palmellopsidaceae & $\begin{array}{l}\text { Chlamydocapsa bacillus (Teiling) Fott } 1972 \\
\text { Chlamydocapsa planctonica (West \& G.S.West) } \\
\text { Fott } 1972\end{array}$ \\
\hline & & Sphaerocystidaceae & $\begin{array}{l}\text { Sphaerocystis schroeteri Chodat } 1897 \\
\text { Sphaerocystis sp. Chodat, } 1897\end{array}$ \\
\hline & & Goniaceae & Gonium formosum Pascher 1927 \\
\hline & & Haematococcaceae & Haematococcus lacustris Wille in Warming, 1884 \\
\hline & & Phacotaceae & Phacotus angustus Pascher 1927 \\
\hline
\end{tabular}




\begin{tabular}{|c|c|c|c|}
\hline Clase & Orden & Familia & Género \\
\hline \multirow[t]{6}{*}{ Cyanophyceae } & Chlamydomonadales & Chlorococcaceae & $\begin{array}{l}\text { Chlorococcum sp. Meneghini, } 1842 \\
\text { Tetraedron minimun (A.Braun) Hansgirg } 1888\end{array}$ \\
\hline & Chlorococcales & Hydrodictyaceae & $\begin{array}{l}\text { Pediastrum duplex Meyen } 1829 \\
\text { Pediastrum simplex Meyen } 1829\end{array}$ \\
\hline & Sphaeropleales & Radiococcaceae & $\begin{array}{l}\text { Coenococcus sp. Korshikov, } 1953 \\
\text { Gloeocystis sp. Nägeli, } 1849\end{array}$ \\
\hline & Ulotrichales & Ulotrichaceae & Ulotrix sp. Kützing, 1833 \\
\hline & Chlamydomonadales & Chlamydomonadaceae & $\begin{array}{l}\text { Chlamydomona docapsa Ehrenberg, } 1833 \\
\text { Chlamydomona sp. Ehrenberg, } 1833\end{array}$ \\
\hline & Volvocales & Volvocaceae & $\begin{array}{l}\text { Eudorina elegans Ehrenberg } 1832 \\
\text { Volvox sp. Linnaeus, } 1758\end{array}$ \\
\hline \multirow[t]{2}{*}{ Trebouxiophyceae } & Chlorellales & Chlorellaceae & Chlorella sp. Beyerinck [Beijerinck], 1890 \\
\hline & Trebouxiales & Botryococcaceae & Botryococcus sp. Kützing, 1849 \\
\hline Dinoflagellata & Peridiniales & Peridiniaceae & Peridinium sp. Ehrenberg, 1830 \\
\hline Euglenophyta & Euglenales & Euglenophycea & $\begin{array}{l}\text { Chlorella sp. Beyerinck [Beijerinck], } 1890 \\
\text { Botryococcus sp. Kützing, } 1849\end{array}$ \\
\hline \multirow[t]{5}{*}{ Bacillariophyceae } & Thalassiophysales & Fragilariaceae & $\begin{array}{l}\text { Meridion sp. C.Agardh, } 1824 \\
\text { Diatoma sp. Bory de St-Vincent, } 1824\end{array}$ \\
\hline & & Naviculaceae & Navicula sp. Bory de Saint-Vincent, 1822 \\
\hline & Aulacoseirales & Aulacoseiraceae & $\begin{array}{l}\text { Aulacoseira granulata (Ehrenberg) Simonsen } \\
1979\end{array}$ \\
\hline & Cymbellales & Cymbellaceae & $\begin{array}{l}\text { Cymbella minuta Hilse in Rabenhorst } 1862 \\
\text { Cymbella cistula (Ehrenberg) O.Kirchner } 1878\end{array}$ \\
\hline & Lithodesmiales & Lithodesmiaceae & Ditylum sp. J.W.Bailey, 1861 \\
\hline
\end{tabular}

Fuente propia

De las especies encontradas durante el estudio, el $59 \%$ corresponde a la clase Cyanophyceae con 48 especies; el $22 \%$ a la clase Chlorophyceae, con 18 especies; $9 \%$ de la clase Bacillariophyceae, con 7 especies; el $4 \%$ la clase Zignematophyceae, con 3 especies; el $3 \%$ la clase Euglenphyceae con 2 especies; el $2 \%$ a la clase Trebouxiophyceae, con 2 especies y el $1 \%$ la clase Dinophyceae, con 1 especie (Cuadro 3). Así como, el porcentaje de los grupos de fitoplancton encontrados en el 
lago de Yojoa, los grupos más representativos en el orden respectivo fueron: cianofitas, clorofitas y bacillariofitas, con un pequeño porcentaje de las familias Trebouxiophyceae, Zygnematophyceae, Dinophyceae y Euglenophyceae.

Reynolds (2006) menciona que el flujo y la disponibilidad de nutrientes son factores importantes que gobiernan la composición y la biomasa del fitoplancton en ecosistemas acuáticos, siendo el nitrógeno y el fósforo los nutrientes que históricamente han sido señalados como los principales factores limitantes para el crecimiento algal.

Es importante mencionar que, la presencia de algas verdeazules en la capa superficial está relacionada con las temperaturas cálidas y este lago ha sido clasificado como cálido tropical monomíctico por Sandoval en 2003 (Studer, 2007) y en el 2006 (Otero, 2011).

\section{Cuadro 3. Grupos taxonómicos del fitoplancton encontrados en el lago de Yojoa, mayo de 2014 a marzo de 2015}

\begin{tabular}{|l|l|l|l|l|l|l|}
\hline \multicolumn{1}{|r}{ Phylum } & \multicolumn{1}{|c|}{ Clase } & Orden & Familia & Género & Especie & $\%$ \\
\hline Cyanobacteria & Cyanophyceae & 4 & 9 & 21 & 48 & 59 \\
\hline Euglenozoa & Euglenophyceae & 1 & 1 & 2 & 2 & 3 \\
\hline Dinoflagelata & Dinophyceae & 1 & 1 & 1 & 1 & 1 \\
\hline Ochrophyta & Bacillariophyceae & 4 & 5 & 6 & 7 & 9 \\
\hline Chlorophyta & Chlorophyceae & 8 & 14 & 17 & 18 & 22 \\
\hline & Trebouxiophyceae & 2 & 2 & 2 & 2 & 2 \\
\hline Charophyta & Zignematophyceae & 1 & 3 & 3 & 3 & 4 \\
\hline & Total & 20 & 32 & 59 & 81 & 100 \\
\hline & & & & & & \\
\hline
\end{tabular}

Fuente propia

Margalef (1983) apunta que el grupo de las cianofitas se desarrolla mejor en aguas alcalinas, tal es el caso del lago de Yojoa, cuyas aguas superficiales presentaron promedios de pH que oscilaron entre 7.5 y 8.2, lo cual crea las condiciones idóneas para el desarrollo de las mismas.

La presencia de algas verdes en las capas superiores de los cuerpos de agua está relacionada con las concentraciones de oxígeno (Scagel y otros, 1987). En el caso 
del lago de Yojoa, el promedio del oxígeno disuelto superficial durante el tiempo de estudio se mantuvo entre 4.06 y $5.34 \mathrm{mg} / \mathrm{l}$, valores que se relacionan de alguna manera con la frecuencia de aparición de las especies de clorofíceas de muy abundantes a abundantes encontradas, como es el caso de Staurastrum leptocladum (0.86), Sphaerocystis schroeteri (0.81). Se presentó una distribución espacial y temporal especialmente abundante al final de la época seca 2014(M1) y la Canícula(M2), muestreos con mayor abundancia de cianofitas. Asimismo, Sphaerocystis sp., Eudorina elegans, Phacotus angustus y otras menos abundantes (ver Cuadro 4).

Round (1981) menciona que las Clorofitas tienden a ser dominantes en lagos tropicales y muy comunes en ambientes eutróficos, como en el caso de Staurastrum leptocladum, especie que siempre estuvo presente espacial y temporalmente en este estudio. Keeble (1996) apunta que esta especie es abundante en hábitats mesotróficos y eutróficos; sin embargo, la mayor abundancia y frecuencia correspondió a las cianofitas, ya que la presencia de materia orgánica en la zona de productividad consume mucho oxígeno, generando incremento de especies indicadoras de contaminación tales como Microcystis aeruginosa 0.92, varias especies del género Microcystis, Aphanocapsa delicatissima 0.89 , Oscillatoria limosa 0.89 y otras más que se enlistan en el Cuadro 4.

Las Bacillariofitas resultaron el tercer grupo con un porcentaje bajo de especies, pero con mucha frecuencia de Aulacoseira granulata en los muestreos de la época lluviosa del 2014 (M3 y M4), y la época seca 2015(M5 y M6). Además esta especie tiene una distribución cosmopolita y junto con algunas de las especies de clorofitas y cianofitas mencionadas anteriormente, han sido reportadas en otros trabajos como especies propias de ambientes eutroficados en lagos tropicales y templados (Robarts y Zohary, 1984; Hornstrom, 1981).

\section{Variación espacial y temporal del fitoplancton}

La variación espacial de la composición de la comunidad fitoplanctónica (Cuadro 5.) se analizó a partir de las especies que tienen una frecuencia de aparición mayor al 40 $\%$ como muy abundantes, entre $20 \%$ - $40 \%$ abundantes, entre $19 \%$ - $6 \%$ menos abundantes y raras entre $6 \%$ - $3 \%$. Del total de 81 especies determinadas en las seis estaciones monitoreadas durante los seis muestreos, 17 resultaron muy abundantes, 9 abundantes, 35 menos abundantes y 20 especies raras.

Espacial y temporalmente se registraron diferencias entre la composición taxonómica y la distribución del fitoplancton entre los diferentes muestreos y estaciones mues- 
treadas durante el estudio, principalmente debido a la presencia, frecuencia, abundancia, dominancia y diversidad de los diferentes grupos encontrados (Hernández y otros, 2008) mencionan que las respuestas del fitoplancton a los cambios ambientales anuales son evidentes. En latitudes tropicales donde la luz y la temperatura son relativamente constantes en el curso del año, las variaciones estacionales dependen de la precipitación y la sequía (Gonzales, 1988), ya que contribuye a los cambios hidrodinámicos del sistema, favoreciendo la circulación de la masa de agua y la generación de numerosas corrientes. Sin duda que es de mucha importancia determinar la frecuencia relativa de la comunidad del fitoplancton en estudios cualitativos durante la época seca y lluviosa, ya que con ello se puede conocer cómo está constituida la comunidad y a su vez, conocer las especies indicadoras y las respuestas de estas a las condiciones ambientales que puedan dar lugar a la sucesión del fitoplancton en ecosistemas acuáticos.

\section{Cuadro 4. Variación de la frecuencia de especies identificadas en el análisis cualitativo en el lago de Yojoa, mayo de 2014 a marzo de 2015}

\begin{tabular}{|c|c|c|c|}
\hline Grupo & Especies & Frecuencia de aparición & Frecuencia relativa \\
\hline \multicolumn{4}{|c|}{ MUY ABUNDANTES } \\
\hline Cyano & Microcystis aeruginosa & 0.92 & 0.41 \\
\hline Cyano & Aphanocapsa delicatissima & 0.89 & 0.40 \\
\hline Cyano & Oscillatoria limosa & 0.89 & 0.40 \\
\hline Zygn & Staurastrum leptocladum & 0.86 & 0.39 \\
\hline Chloro & Sphaerocystis schroeteri & 0.81 & 0.36 \\
\hline Cyano & Aphanocapsa elachista & 0.72 & 0.33 \\
\hline Cyano & Pseudoanabaena mucícola & 0.67 & 0.30 \\
\hline Cyano & Gleocapsa spp. & 0.64 & 0.29 \\
\hline Cyano & Aphanocapsa mucicola & 0.64 & 0.29 \\
\hline Cyano & Dolichospermum solitarium & 0.58 & 0.26 \\
\hline Cyano & Aphanocapsa spp & 0.56 & 0.25 \\
\hline Cyano & Lyngbya sp. & 0.53 & 0.24 \\
\hline Cyano & Chrococcus spp & 0.50 & 0.23 \\
\hline Dino & Peridinium sp. & 0.50 & 0.23 \\
\hline Cyano & Pseudoanabaena galeata & 0.47 & 0.21 \\
\hline Baci & Aulacoseira granulata & 0.44 & 0.20 \\
\hline Cyano & Trichodesmium lacustre & 0.42 & 0.19 \\
\hline
\end{tabular}

Fuente propia 


\begin{tabular}{|c|c|c|c|}
\hline Grupo & Especies & Frecuencia de aparición & Frecuencia elativa \\
\hline \multicolumn{4}{|c|}{ ABUNDANTES } \\
\hline Chloro & Eudorina elegans & 0.36 & 0.16 \\
\hline Cyano & Microcystis spp. & 0.33 & 0.15 \\
\hline Cyano & Phormidium sp. & 0.31 & 0.14 \\
\hline Chloro & Chlamydocapsa plantónica & 0.28 & 0.13 \\
\hline Cyano & Aphanothece spp. & 0.28 & 0.13 \\
\hline Chloro & Phacotus angustus & 0.28 & 0.13 \\
\hline Chloro & Sphaerocystis sp. & 0.25 & 0.11 \\
\hline Cyano & Aphanocapsa incerta & 0.25 & 0.11 \\
\hline Cyano & Aphanocapsa koordersii & 0.22 & 0.10 \\
\hline \multicolumn{4}{|c|}{ MENOS ABUNDANTES } \\
\hline Cyano & Borzia trilocularis & 0.19 & 0.09 \\
\hline Cyano & Oscillatoria prínceps & 0.19 & 0.09 \\
\hline Cyano & Chrococcus limneticus & 0.17 & 0.08 \\
\hline Cyano & Chrococcus dispersus & 0.17 & 0.08 \\
\hline Cyano & Aphanocapsa endophytica & 0.17 & 0.08 \\
\hline Cyano & Pseudanabaena sp. & 0.17 & 0.08 \\
\hline Cyano & Coelosphaerium sp. & 0.17 & 0.08 \\
\hline Chloro & Botryococcus sp. & 0.17 & 0.08 \\
\hline Chloro & Gloeocystis sp. & 0.14 & 0.06 \\
\hline Cyano & Aphanocapsa holsatica & 0.14 & 0.06 \\
\hline Cyano & Dolichospermun circinalis & 0.14 & 0.06 \\
\hline Cyano & Dolichospermum sp. & 0.14 & 0.06 \\
\hline Chloro & Chlamydocapsa Bacillus & 0.14 & 0.06 \\
\hline Chloro & Chlamydomona sp. & 0.14 & 0.06 \\
\hline Cyano & Synechocystis sp. & 0.11 & 0.05 \\
\hline Cyano & Aphanocapsa planktonica & 0.11 & 0.05 \\
\hline Chloro & Chlorococcum sp. & 0.11 & 0.05 \\
\hline Chloro & Ulotrix sp. & 0.11 & 0.05 \\
\hline Eugle & Phacus sp. & 0.11 & 0.05 \\
\hline Cyano & Dolichospermum spiroides & 0.08 & 0.04 \\
\hline Cyano & Microcystis botrys & 0.08 & 0.04 \\
\hline Cyano & Gloeothece sp. & 0.08 & 0.04 \\
\hline Chloro & Volvox sp. & 0.08 & 0.04 \\
\hline Cyano & Microcystis potocystis & 0.06 & 0.03 \\
\hline Cyano & Asterocapsa submersa & 0.06 & 0.03 \\
\hline Cyano & Oscillatoria nigriviridis & 0.06 & 0.03 \\
\hline Cyano & Oscillatoria germinata & 0.06 & 0.03 \\
\hline
\end{tabular}




\begin{tabular}{|c|c|c|c|}
\hline Grupo & Especies & Frecuencia de aparición & Frecuencia elativa \\
\hline Cyano & Oscillatoria sancta & 0.06 & 0.03 \\
\hline Cyano & Lyngbya spirulinoidea & 0.06 & 0.03 \\
\hline Chloro & Tetraedron mínimum & 0.06 & 0.03 \\
\hline Chloro & Pediastrum dúplex & 0.06 & 0.03 \\
\hline Chloro & Pediastrum simples & 0.06 & 0.03 \\
\hline Chloro & Chlorella sp. & 0.06 & 0.03 \\
\hline Zygn & Cosmarium minutis & 0.06 & 0.03 \\
\hline Zygn & Tribonema sp. & 0.06 & 0.03 \\
\hline \multicolumn{4}{|c|}{ RARAS } \\
\hline Cyano & Microcystis flosaquae & 0.03 & 0.01 \\
\hline Cyano & Oscillatoria sp. & 0.03 & 0.01 \\
\hline Cyano & Oscillatoria ornata & 0.03 & 0.01 \\
\hline Cyano & Lyngbya virgey & 0.03 & 0.01 \\
\hline Cyano & Synechococcus nidulans & 0.03 & 0.01 \\
\hline Cyano & Planktothrix sp. & 0.03 & 0.01 \\
\hline Cyano & Leptolynghbya perelegans & 0.03 & 0.01 \\
\hline Cyano & Limnotrix sp. & 0.03 & 0.01 \\
\hline Cyano & Limnococcus sp. & 0.03 & 0.01 \\
\hline Chloro & Gonium formusum & 0.03 & 0.01 \\
\hline Chloro & Haematococcus lacustris & 0.03 & 0.01 \\
\hline Chloro & Coenococcus sp & 0.03 & 0.01 \\
\hline Chloro & Chlamydomona docapsa & 0.03 & 0.01 \\
\hline Eugl & Euglena sp. & 0.03 & 0.01 \\
\hline Baci & Meridion sp. & 0.03 & 0.01 \\
\hline Baci & Diatoma sp. & 0.03 & 0.01 \\
\hline Baci & Navicula sp. & 0.03 & 0.01 \\
\hline Baci & Cymbella minuta & 0.03 & 0.01 \\
\hline Baci & Cymbella cistula & 0.03 & 0.01 \\
\hline Baci & Ditylum sp. & 0.03 & 0.01 \\
\hline
\end{tabular}

Para comprender la distribución espacial y temporal del fitoplancton en el estudio, se realizó un análisis de las especies por estación, en el cual se pone en evidencia lo siguiente:

1. Estación 1: estación localizada en la desembocadura del río Varsovia al sur del lago de Yojoa, se reportaron 42 de las 81 especies encontradas durante el estudio. De las 48 especies, 28 son cianobacterias y 12 Clorofíceas. Dos especies de las cianofíceas (Dolichospermun spiroides y Oscilatoria germinata) solo 
se encontraron en esta estación de muestreo. Oscillatoria sancta y Lyngbya spirulinoidea únicamente se encuentran en esta estación y en la 2. La clorofícea Tribonema sp. solo estuvo presente en esta estación y la Cosmarium minutis solo se encontró en las estaciones de muestreo 1 y 2.

2. Estación 2: ubicada en la desembocadura de la quebrada Jutiapa, entre las estaciones 1 y 3 . Aquí se encontraron 50 especies de las 81 reportadas; de estas especies, 33 pertenecen a las cianoficeas y 13 a las Cloroficeas. Solamente en la estación 6 se encontraron más especies que las reportadas en esta estación. Además de las especies de cianofitas y clorofitas halladas también en la estación 1, las especies Oscilatoria sp. y Limnococcus sp. solamente se encontraron en esta estación, al igual que la Clorofita Haematococcus lacustris y la Diatomea Navicula sp.

3. Estación 3: denominada punto índice (más o menos en el centro del lago), las 100 o más jaulas de cultivo de peces de la Empresa Aquafinca Saint Peter Fish se encontraban en las cercanías de la estación de muestreo hacia el este, durante los tres primeros muestreos, pero en los siguientes muestreos quedamos en el centro, lo que indica que las jaulas son movidas continuamente, posiblemente para que los desechos de comida y excretas de peces y humanos no se acumulen tanto que afecten la calidad del agua. Para esta estación se reportó el menor número de especies (40) en todo el estudio, 27 cianofitas y 9 clorofitas. Asimismo, se reportó la diatomea Meridion sp. que aparentemente solo apareció en esta estación de muestreo. La baja diversidad encontrada en este sitio indica que la actividad realizada por la empresa antes mencionada afecta sustantivamente la calidad del agua. Durante el estudio, en esta estación apareció el mayor número de células $(82,000)$ / $\mathrm{ml}$ de Aphanocapsa delicatissima, específicamente durante la Canícula (M2).

4. Estación 4: situada en la desembocadura de la quebrada Raíces, mejor conocida como la quebrada Cianuro, se encontraron 45 especies, de las 81 reportadas para todo el estudio. 30 de ellas pertenecen a las Cianofitas y 9 especies a las clorofitas. Las cianofitas Synechococcus nidulans, Oscillatoria nigriviridis y Lyngbya virgey solamente se encontraron en esta estación. Sin embargo, Microcystis potocystis únicamente se encontró en esta estación y en la estación 5. También las diatomeas Diatoma sp, Cymbella minuta y Cymbella cistula solamente se encontraron en esta estación. La mayor cantidad de células $/ \mathrm{ml}$ de Microcystis aeruginosa $(148,333)$ se encontró en esta estación durante el primer muestreo (M1) el 30 de mayo de 2014. 
5. Estación 5: denominada río Helado, se encuentra en la desembocadura del río Blanco y está contiguo al canal de drenaje hacia la presa de Cañaveral. Aquí se reportaron 45 especies, de las 81 reportadas para todo el lago; 30 de ellas pertenecen a las cianofitas y 11 a las clorofitas. De las cianofíceas solamente se reportaron para esta estación: Microcystis flosaquae, Asterocapsa submersa y Oscillaroria ornata. Comparte la aparición de Mycrocystis botrys y Microcystis potocystis con las estaciones vecinas solamente. De las clorofíceas, Gonium formosum solo se registró para esta estación.

6. Estación 6: localizada frente al Hotel Brisas del Lago. En esta estación se reportó la mayor parte de especies (52), de las cuales 33 son cianofitas y 14 son clorofitas. Las especies compartidas con otras estaciones fueron Microcystis botrys (con la estación 5) y Oscillatoria nigriviridis (con la estación 4). De las especies que solamente aparecieron en esta estación, Planktothrix sp., Leptolyngbya perelegans y Limnotrix sp. pertenecen a las cianofíceas; Tribonema sp., Tetraedron minimun, Pediastrum duplex, Pediastrum simplex, Chlamydomona docapsa y Chlorella sp. del grupo de las cloroficeas; Euglena sp. pertenece a las Euglenales y Ditylum sp. a las bacilarioficeas. En resumen, espacialmente este lugar presentó la mayor riqueza específica.

Es muy posible que la aparición de algunas especies esté determinada por las algas que se desarrollan en cada sistema lótico que aporta sus aguas al lago de Yojoa. Este sería un tema a abordar en otros estudios relacionados con el fitoplancton y las particularidades del lago de Yojoa (corrientes prevalecientes de las masas de agua con el manejo de las descargas para la Represa Cañaveral, corrientes internas, corriente superficiales, etc.). Igualmente, la morfología del lago, el clima y parámetros como luz y temperatura, la forma y disponibilidad de los nutrientes para el fitoplancton, podrían influenciar la distribución de las cianobacterias en el lago.

En lagos tropicales profundos se sabe que hay un patrón en la sucesión biológica, influenciada por la hidrodinámica de la columna de agua y eventos meteorológicos (Hernández y otros, 2011), los cuales crean condiciones específicas para el florecimiento de diferentes grupos algales. De León y Chalar (2003) mencionan que la comunidad fitoplanctónica se estructura básicamente con relación a la disponibilidad de nutrientes y luz. Sin embargo, la tasa de crecimiento fitoplanctónico depende de 3 componentes principales: temperatura, radiación solar y nutrientes, siendo el factor más influyente la luz (CEPIS, 1996). De León y Chalar (2003) consideran que en ambientes con disponibilidad de nutrientes, predominan organismos oportunistas, de rápido crecimiento y reproducción, generalmente de pequeña y alta relación superfi- 
cie/volumen, como los fitoflagelados. En ambientes con limitación de nutrientes, predominan los organismos tolerantes al estrés, como las cianofitas.

En este estudio se puede observar desde el inicio de los muestreos (Cuadro 5) que la frecuencia y abundancia siguió el patrón siguiente: en primer lugar, cianofitas; en segundo lugar, las clorofitas y en tercer lugar, las bacillariofitas, siguiendo otros grupos, pero en menor frecuencia y abundancia, concluyendo que de las Ciaonofitas fueron frecuentes estas: Microcystis aeruginosa y Microcystis sp. en los muestreos M1 y M4; en los muestreos M2, M3, M5 y M6 la más frecuente y abundante fue Aphanocapsa delicatissima.

Cuadro 5. Presencia de especies de fitoplancton por grupo taxonómico por estación y muestreo con el análisis cualitativo en el lago de Yojoa, mayo de 2014 a marzo de 2015

\begin{tabular}{|c|c|c|c|c|c|c|c|}
\hline Estaciones & Grupos & M1 & M2 & M3 & M4 & M5 & M6 \\
\hline \multirow{7}{*}{1} & Cyano & 7 & 10 & 11 & 15 & 13 & 9 \\
\hline & Zygm & 1 & 3 & - & - & 1 & 1 \\
\hline & Chlor & 1 & 1 & 3 & 5 & 4 & 3 \\
\hline & Dino & - & 1 & 1 & 1 & 1 & 1 \\
\hline & Eugl & - & - & - & - & - & - \\
\hline & Baci & - & - & - & 1 & 1 & - \\
\hline & Total & 9 & 15 & 15 & 22 & 20 & 14 \\
\hline \multirow{7}{*}{2} & Cyano & 11 & 15 & 13 & 15 & 12 & 16 \\
\hline & Zygm & 2 & 1 & - & 1 & 1 & 1 \\
\hline & Chlor & 3 & 1 & 3 & 3 & 4 & 4 \\
\hline & Dino & - & - & - & 1 & 1 & 1 \\
\hline & Eugl & 1 & - & - & - & - & - \\
\hline & Baci & - & - & - & 1 & 1 & - \\
\hline & Total & 17 & 17 & 16 & 21 & 19 & 22 \\
\hline
\end{tabular}




\begin{tabular}{|c|c|c|c|c|c|c|c|}
\hline Estaciones & Grupos & M1 & M2 & M3 & M4 & M5 & M6 \\
\hline \multirow{7}{*}{3} & Cyano & 11 & 8 & 16 & 9 & 8 & 14 \\
\hline & Zygm & 1 & 1 & - & 1 & 1 & 1 \\
\hline & Chlor & 3 & 4 & 5 & 2 & 2 & 2 \\
\hline & Dino & - & 1 & 1 & - & 1 & 1 \\
\hline & Eugl & - & - & - & - & - & - \\
\hline & Baci & 1 & - & - & 1 & 1 & - \\
\hline & Total & 16 & 14 & 22 & 13 & 13 & 18 \\
\hline \multirow{7}{*}{4} & Cyano & 9 & 18 & 16 & 14 & 11 & 15 \\
\hline & Zygm & 1 & 1 & - & 1 & 1 & 1 \\
\hline & Chlor & 3 & 2 & 2 & 3 & 3 & 5 \\
\hline & Dino & - & - & - & - & 1 & - \\
\hline & Eugl & - & - & - & - & 1 & - \\
\hline & Baci & - & 1 & - & 1 & 1 & 3 \\
\hline & Total & 13 & 22 & 18 & 19 & 18 & 24 \\
\hline \multirow{7}{*}{5} & Cyano & 12 & 15 & 16 & 10 & 11 & 16 \\
\hline & Zygm & 1 & 1 & 1 & 1 & 1 & 1 \\
\hline & Chlor & 3 & 1 & 2 & 2 & 3 & 4 \\
\hline & Dino & - & - & - & 1 & - & - \\
\hline & Eugl & - & - & - & - & - & 1 \\
\hline & Baci & - & - & - & 1 & 1 & 1 \\
\hline & Total & 16 & 17 & 19 & 15 & 16 & 23 \\
\hline
\end{tabular}




\begin{tabular}{|c|c|c|c|c|c|c|c|}
\hline Estaciones & Grupos & M1 & M2 & M3 & M4 & M5 & M6 \\
\hline \multirow{7}{*}{6} & Cyano & 13 & 17 & 16 & 14 & 14 & 16 \\
\hline & Zygm & 1 & 2 & 1 & 1 & 1 & 1 \\
\hline & Chlor & 2 & 7 & 4 & 3 & 3 & 2 \\
\hline & Dino & 1 & - & 1 & 1 & 1 & - \\
\hline & Eugl & - & - & - & 1 & 1 & 1 \\
\hline & Baci & - & 1 & 1 & 1 & 1 & 1 \\
\hline & Total & 17 & 27 & 23 & 21 & 21 & 21 \\
\hline
\end{tabular}

Fuente propia

\section{Abundancia de la comunidad fitoplanctónica}

De acuerdo con los resultados, la abundancia de la comunidad fitoplanctónica analizada basándose en el número de células/especie/ $\mathrm{ml}$ (biovolumen), resultó de la manera siguiente: el primer muestreo al final de la época seca 20014(M1) con $351,693 \mathrm{cel} / \mathrm{ml}$, de las cuales Microcystis aeruginosa obtuvo 155,001 células/ml distribuidas en las estaciones Río Varsovia (E1), Quebrada Jutiapa entre la Puntilla y el Cacao (E2) y Punto índice (E3) y Microcystis sp. con 192,466 cel/ml distribuidas en las estaciones de Quebrada Cianuro (E4), Rio Helado (E5) y la estación frente a Hotel Brisas del Lago (E6); Oscillatoria limosa en las estaciones E1, E2 y E6 con 2001 $\mathrm{cel} / \mathrm{ml}$ y Sphaerocystis Schroeteri con $2052 \mathrm{cel} / \mathrm{ml}$ distribuidas en las 6 estaciones.

En el segundo muestreo durante la Canícula (M2) con un total de 197,106 cel/ml, de las cuales aparecieron Aphanocapsa delicatissima con 188,361 cel/ml presentes en todas las estaciones (E1- E6), resultando con mayor abundancia en la estación E3 con 82,000 cel/ml, Sphaerocystis schroeteri con $6640 \mathrm{cel} / \mathrm{ml}$ distribuidas en las estaciones E1, E3, E5 y E6; siendo la estación E3 (punto índice) la que obtuvo el mayor número de células $(5120 \mathrm{cel} / \mathrm{ml})$. En el tercer muestreo durante la época lluviosa (M3) un total de 49,671 cel/ml, de las cuales 46,467 cel/ml corresponden a Aphanocapsa delicatissima, distribuidas en las estaciones E1, E2, E3 y E6; siendo más abundantes en la estación E1 con 15,853 cel/ml y en la estación E6 con 13,667 cel/ml y Aphanocapsa incerta con $1773 \mathrm{cel} / \mathrm{ml}$ en las estaciones E1, E4, E5 y E6.

En el cuarto muestreo al final de la época lluviosa e inicios de la época seca (M4) un 
total de 23,519 cel/ml, de las cuales 23,332 cel/ml corresponden a Microcystis sp. con presencia en todas las estaciones (E1-E6), siendo más abundantes en la estación E6 con $6667 \mathrm{cel} / \mathrm{ml}$ y Gleocapsa sp. con $187 \mathrm{cel} / \mathrm{ml}$ distribuidas en las estaciones E1, E2, E3, E4 y E6. En el quinto muestreo en la época seca del 2015 (M5), se reportaron $11,178 \mathrm{cel} / \mathrm{ml}$, de las cuales $7653 \mathrm{cel} / \mathrm{ml}$ corresponden a Aphanocapsa delicatissima distribuidas en las estaciones E1, E2, E3, E4 y E5, siendo más abundantes en E5 con $2187 \mathrm{cel} / \mathrm{ml}$ y Oscillatoria limosa con $2001 \mathrm{cel} / \mathrm{ml}$ distribuidas en la E1 con $1334 \mathrm{cel} / \mathrm{ml}$ y en la E3 con $667 \mathrm{cel} / \mathrm{ml}$. También estuvieron presentes Trichodesmium lacustre con $1334 \mathrm{cel} / \mathrm{ml}$ con presencia en E3 y E6. En el sexto muestreo durante la época seca en Marzo de I2015 (M6) aparecieron 10,044 cel/ml, siendo la especie más abundante Aphanocapsa delicatissima en las estaciones E1, E2, E4 y E5 con $5834 \mathrm{cel} / \mathrm{ml}$ y Aphanocapsa elachista en las E2 con E4, E5 y E6 con $1340 \mathrm{cel} / \mathrm{ml}$ y Lyngbya sp. en E2 con $2000 \mathrm{cel} / \mathrm{ml}$.

Al analizar los resultados anteriores se puede evidenciar la floración de Microcystis aeruginosa en el primer muestreo (M1), específicamente en las estaciones E1, E2 y E3 y la floración de Aphanocapsa delicatissima en el segundo muestreo durante la Canícula (M2) en las estaciones E1, E2, E3 y E4, con presencia abundante de Aphanocapsa delicatissima y Aphanocapsa incerta, pero no en los niveles que se consideren como afloramiento. Lo mismo sucedió al final de época lluviosa (M4) con abundancia de cel/ml para Microcystis sp. como se menciona anteriormente. Los datos anteriores coinciden con el patrón de lluvia en el lago (figura 2), lo que pudo haber arrastrado cantidades considerables de fósforo, nitrógeno y otras sustancias que afectan el pH básico del agua superficial, turbidez y transparencia.

Bajo ciertas condiciones, especialmente en donde las aguas son ricas en nutrientes con aumento en el contenido de fósforo y nitrógeno (cuadro 1) provenientes principalmente de actividades agrícolas y de efluentes cloacales y expuestas a radiación solar, las cianobacterias pueden multiplicarse hasta alcanzar altas densidades. Cuando esta proliferación es dominada por una especie o por algunas pocas, dicho fenómeno se conoce como Floración algal (Conti y otros, 2005). Con relación al promedio por muestreo se puede observar en el cuadro 6 , figura 3, que el número de células fue disminuyendo progresivamente durante el período de estudio, encontrándose la mayor cantidad de células/ml en M1 $(58,616)$ y la menor cantidad (1674) en M6.

De ese resultado se puede evidenciar la abundancia promedio de las cianobacterias en M1, que coincidió con el final de un evento de floración algal. Es posible que esta floración haya ocurrido a mediados del mes de mayo de 2014, ya que al momento del 
primer muestreo se observó una nata superficial de coloración café verdoso correspondiente a algas filamentosas del género Oscillatoria limosa y Lyngbya sp. y al momento de la identificación se observó abundancia de fitoflagelados y vorticelas entre las colonias de Microcystis aeruginosa.

Figura 3. Variación del promedio por muestreo del biovolumen en las diferentes estaciones durante los diferentes muestreos (b) en el lago de Yojoa del 30 de mayo de 2014 al 23 de marzo de 2015

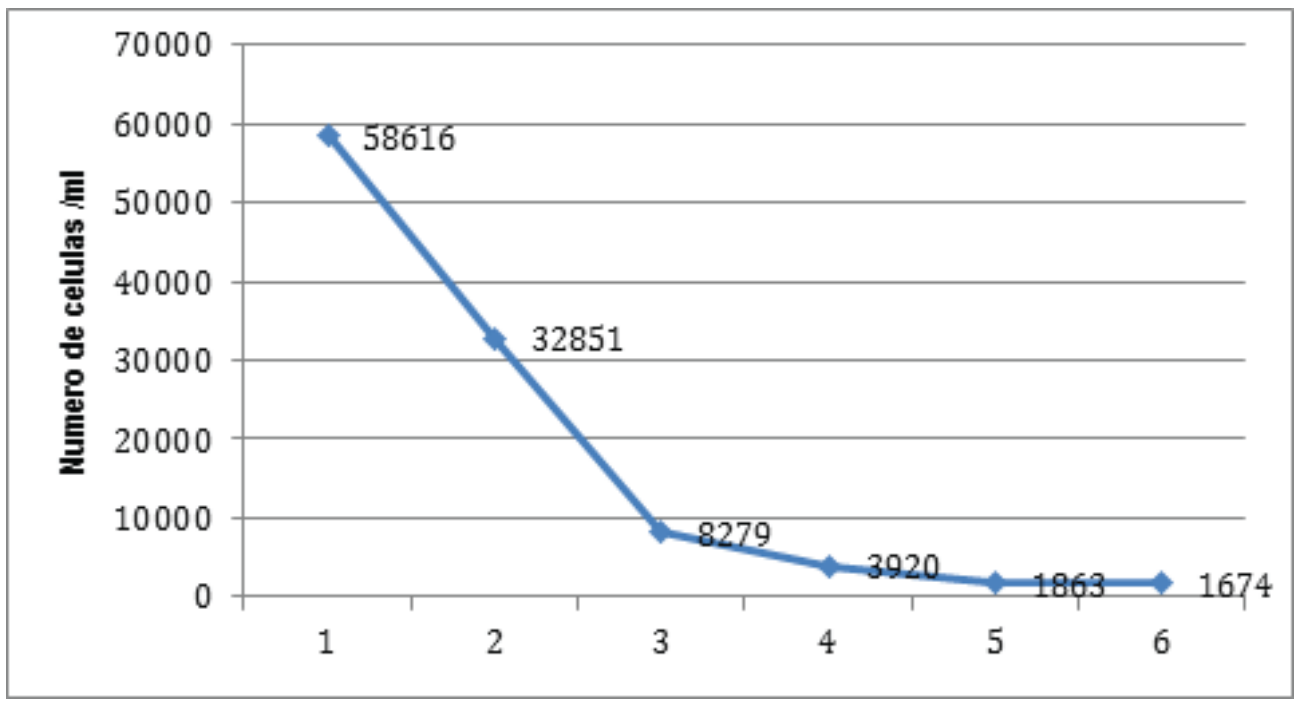

Fuente propia

Normalmente las comunidades en proceso de eutroficación o eutroficadas tienen abundancia de especies y baja diversidad. Margalef (1983) apunta que cualquier aceleración en los procesos del ecosistema se refleja en una disminución del valor numérico de la diversidad, así que es común que los lagos eutroficados y muy eutroficados tengan muy baja diversidad de la comunidad del fitoplancton. La marcada estacionalidad de abundancia y biomasa del fitoplancton y las características ambientales son los factores determinantes de las diferencias temporales en la diversidad encontrada en el ecosistema.

Según Pizzolon (1996), los procesos de eutroficación producen cambios cuantitativos y cualitativos en la comunidad fitoplanctónica, aumentando la frecuencia y densidad de floraciones y se observan comportamientos en donde las cianobacterias desplazan a las diatomeas, clorofíceas o crisofíceas. En ambientes altamente eutroficados, las cianobacterias suelen predominar durante la mayor parte del año. Hechos que han sido verificados en muchos trabajos limnológicos, formulándose diversas hipótesis al respecto. 


\section{Cuadro 6. Variación del número de células por ml (biovolumen en el lago de Yojoa del 30 de mayo de 2014 al 23 de marzo de 2015}

\begin{tabular}{|c|c|c|c|c|c|c|c|}
\hline \multicolumn{8}{|c|}{ BIOVOLUMEN (Cel/ml) } \\
\hline Estación & Muestreo 1 & Muestreo 2 & Muestreo 3 & Muestreo 4 & Muestreo 5 & Muestreo 6 & Promedio \\
\hline $\mathrm{E} 1$ & 62947 & 32924 & 15940 & 3400 & 3011 & 1330 & 19,925 \\
\hline E2 & 57574 & 23523 & 10450 & 3373 & 1667 & 4766 & 16,892 \\
\hline E3 & 37340 & 87126 & 7677 & 3360 & 2430 & 2017 & 23,325 \\
\hline E4 & 148413 & 21867 & 400 & 3373 & 1210 & 1954 & 29,536 \\
\hline E5 & 18896 & 20149 & 330 & 3333 & 2187 & 1557 & 7,742 \\
\hline E6 & 26523 & 11517 & 14874 & 6680 & 673 & 920 & 10,198 \\
\hline Total & 351,693 & 197,106 & 46,671 & 23,519 & 11,178 & 10,044 & \\
\hline Prom & 58,616 & 32,851 & 8,279 & 3,920 & 1,863 & 1,674 & \\
\hline $\operatorname{Max}$ & 148,413 & 87,126 & 15,940 & 6,680 & 3,011 & 4,766 & \\
\hline Min & 26,523 & 11,517 & 330 & 3,333 & 673 & 920 & \\
\hline
\end{tabular}

Fuente propia

El predominio de las cianobacterias se explicaría en todos los casos por su elevada capacidad para absorber bióxido de carbono disuelto, aún en concentraciones muy bajas; el pH elevado favorece el desarrollo de las cianobacterias, por su capacidad para transformar los iones bicarbonato y carbonato en bióxido de carbono. De hecho, las cianobacterias son las únicas algas que desarrollan biomasas importantes en ambientes naturalmente alcalinos y salinos. En cuerpos de agua someros su concentración suele aumentar durante los años secos.

Es importante hacer notar que Studer (2007) ya había registrado, en un estudio en el lago de Yojoa, la presencia de abundantes cianobacterias (Microcystis sp. y Oscilatoria sp.), posiblemente durante un florecimiento algal, indicando además la presencia de 13 géneros de fitoplancton, sin embargo, no menciona cuales son, pero indica que de los géneros mencionados anteriormente, estos representaban el $70 \%$ de la totalidad. La ocurrencia del evento coincide con la ocurrencia de floración algal de este estudio. 
Variación en la diversidad, dominancia y similitud espacial y temporal del fitoplancton del lago de Yojoa durante el periodo de estudio

Margalef (1997) afirma que las variaciones de la diversidad están asociadas a las características productivas de la comunidad. La marcada temporalidad y espacialidad de la abundancia y biomasa del fitoplancton y de las características ambientales estudiadas, serían los factores que determinan las diferencias espaciales y temporales en la diversidad encontrada en el sistema. Igualmente, menciona que la disminución de la diversidad indica un aumento en la actividad metabólica de los organismos, de modo que las variaciones de diversidad están asociadas a las características productivas de la comunidad.

La diversidad de la comunidad biológica se ve fuertemente afectada por los cambios en el ciclo hidrológico que modifica casi todos los parámetros fisicoquímicos, a la vez que tiene fuerte influencia en el fitoplancton, siendo las microalgas planctónicas los productores primarios del sistema y, por lo tanto, la base de la pirámide trófica; cualquier cambio que afecte su distribución y abundancia afecta al resto de organismos del sistema que también sufren de manera directa dichos cambios (Prosperi, 1999 y 2000).

Dentro de un ciclo anual, las condiciones de un lago se modifican siguiendo un patrón regular, el cual condiciona que las comunidades algales estén sometidas a una sucesión estacional (Novoa y otros, 2011). También expresan que el origen del sistema léntico, el tamaño, forma y superficie del espejo de agua, profundidad, naturaleza del fondo, radiación solar, estado trófico y otras variables fisicoquímicas, son factores que influyen sobre el crecimiento y desarrollo de distintas especies algales y sus variaciones temporales. Los cambios en la diversidad y la química del agua para un lago se han atribuido a una progresiva eutrofización asociada al uso y a una intensa actividad humana (Ridying \& Rast, 1992; Gonzales y otros, 2003).

Novoa y otros (2011) mencionan que es conocido que la variación temporal del fitoplancton está relacionada con la fluctuación de los factores ambientales, como es el caso de la temperatura y la transparencia que afectan, en mayor grado, la distribución espacial del fitoplancton y, a su vez, la fuente de luz recibida determina un patrón de variabilidad espacial y temporal.

El índice de Whittaker para la diversidad $\beta$ para el lago de Yojoa en el período 2014-2015, utilizando un número total de especies (81) y promedio de especies por muestreo (18), fue de 4.76. De acuerdo con los resultados, la mayor riqueza específica (10.58) fue en la estación frente al Hotel Brizas del Lago (E6) y la menor (8.56) en 
la estación Punto Índice (E3). Temporalmente, la mayor riqueza específica fue de 9.98 en el segundo muestreo durante la Canícula (M2) y la menor de 7.28 en la época seca en Enero 2015 (M5). Los demás resultados de los índices de riqueza específica, espacial y temporal, fueron muy semejantes entre ellos. De León y Chalar (2003) menciona que: "Los mejores indicadores de los cambios en las características tróficas de cuerpos de agua lénticos y lóticos serían las variaciones en la composición y abundancia del fitoplancton, más que los índices de diversidad" (Pag.110).

El índice de dominancia específica de Berger-Parker mide la dominancia del taxón más abundante. Valores entre 0 y $1(0 \%$ y 100\%) indica polución orgánica, degradación en la morfología del cuerpo de agua y degradación general. Se traduce como una disminución en la equidad y un aumento en la dominancia. Mientras más se acerca a 1, habrá más dominancia de una especie y menos equidad en la distribución y representación de los organismos. Según De León y Chalar (2003), los lagos dominados por cianobacterias son eutróficos, ya que bajo ciertas condiciones, especialmente donde las aguas son ricas en nutrientes y expuestas a la luz solar, estas pueden multiplicarse hasta alcanzar altas densidades.

Durante el estudio, el índice de dominancia espacial muestra que la mayor dominancia (0.38) corresponde a la estación de la Quebrada Cianuro(E4). Las especies dominantes en esta estación fueron: Microcystis aeruginosa, Aphanocapsa delicatissima, Aphanocapsa elachista, Aphanothece spp, Oscillatoria limosa, Pseudoanabaena mucícola, Staurastrum leptocladum y Sphaerocystis schroeteri. La menor dominancia (0.24) fue en la estación Punto Indice (E3) con las especies: Microcystis aeruginosa, Aphanocapsa delicatissima, Oscillatoria limosa y Staurastrum leptocladum.

La mayor dominancia temporal fue de 0.43 en la época de la Canícula (M2)dominando las especies: Microcystis aeruginosa, Aphanocapsa delicatissima, Aphanocapsa elachista, Aphanothece spp., Oscillatoria limosa, Staurastrum leptocladum y Sphaerocystis schroeteri. El valor más bajo de la dominancia fue de 0.27 al final de la época lluviosa (M4). Las especies que dominaron durante el tercer muestreo fueron: Dolichospermun solitarium, Gleocapsa spp., Aphanocapsa delicatissima, Aphanocapsa elachista y Oscillatoria limosa; durante el cuarto muestreo las especies dominantes fueron: Microcystis aeruginosa, Gleocapsa spp., Aphanocapsa delicatissima, Oscillatoria limosa y Aulacoseira granulata. No hubo diferencia muy significativa en la dominancia de especies en las demás estaciones y muestreos.

De acuerdo a los resultados del índice de similitud espacial, se encontró que la mayor similitud (0.68) fue entre la Puntilla y el Cacao (E2) y la estación Punto Indice (E3) y 
el menor índice (0.48) entre la estación del Rio Varsovia (E1 y la estación del Rio Cianuro (E4). En cambio, la similitud temporal fue mayor (0.74) entre el verano de enero 2015(M5) y el muestro de Marzo del mismo año (M6); la menor (0.33) entre el muestreo de la Canícula (M2) y el muestreo de la época seca del 2015(M5). Al comparar los índices de similitud espacial se encontró que las estaciones vecinas E1-E2, E2-E3, E3-E5 y E5-E6 presentan índices relativamente altos. Asimismo, las estaciones que son influenciadas directamente por la desembocadura de cuerpos de agua corresponden a E1-E5 y E2-E5. Sin embargo, temporalmente la mayor similitud resultó entre el M5 y M6 (0.74); la menor similitud entre la época seca 2014 (M1) y la época lluviosa (M3) y entre la Canícula (M2) y la época seca del 2015 (M5) con un valor de 0.34 .

Con base en el análisis de varianza (ANOVA), el resultado correspondió a 0.77 y la probabilidad es mayor de 0.05 , por lo que la hipótesis nula se acepta, ya que el promedio de organismos entre muestreos (épocas del año) es igual, con un $95 \%$ de confiabilidad y como hipótesis alterna nos dice que al menos en un muestreo el promedio de organismos es distinto, con 95\% de confiabilidad.

\section{CONCLUSIONES Y OBSERVACIONES}

El fitoplancton presente en el Lago de Yojoa estuvo representado en el estudio por 81 especies divididas en 7 clases así: Cyanophyceae, Chlorophyceae, Bacillariophyceae, Zignematophyceae, Euglenophyceae, Trebouxiophyceae y Dinophyceae; de las cuales corresponden el 59\% a la clase Cyanophyceae, con 48 especies; el $22 \%$ a la clase Chlorophyceae, con 18 especies; $9 \%$ de la clase Bacillariophyceae, con 7 especies; el $4 \%$ la clase Zignematophyceae, con 3 especies; el 3\% la clase Euglenphyceae con 2 especies, el $2 \%$ a la clase Trebouxiophyceae, con 2 especies y el $1 \%$ la clase Dinophyceae, con 1 especie.

Todos los análisis relacionados con la composición, la riqueza de especies y la diversidad del fitoplancton indican que la diversidad del fitoplancton del lago de Yojoa es relativamente alta (diversidad beta en todo el lago), pero el grupo de las cianoficeas dominaron la comunidad del fitoplancton, independientemente de la época y de la localización de las estaciones de muestreo.

Espacialmente y temporalmente se registraron diferencias entre la composición taxonómica y la distribución del fitoplancton, principalmente debido a la presencia, 
frecuencia, abundancia, dominancia y diversidad de los diferentes grupos encontrados.

Durante el estudio, la mayor dominancia espacial corresponde a la estación Quebrada Cianuro ó Quebrada Raíces(E4). Las especies dominantes en esta estación fueron: Microcystis aeruginosa, Aphanocapsa delicatissima, Aphanocapsa elachista, Aphanothece spp., Oscillatoria limosa, Pseudoanabaena mucícola, Staurastrum leptocladum y Sphaerocystis schroeteri. La menor dominancia fue en la estación Punto índice donde se encuentran la Jaulas de Aquafinca Saint Peter Fish (E3), con las especies: Microcystis aeruginosa, Aphanocapsa delicatissima, Oscillatoria limosa y Staurastrum leptocladum. En lo que respecta a la dominancia temporal, el mayor valor correspondió a la época lluviosa (canícula), en donde las especies dominantes fueron: Microcystis aeruginosa, Aphanocapsa delicatissima, Aphanocapsa elachista, Aphanothece spp., Oscillatoria limosa, Staurastrum leptocladum y Sphaerocystis schroeteri. La menor dominancia se obtuvo durante la época lluviosa (M3) y al inicio de la época seca (M4). Sin embargo, las especies que dominaron durante la época lluviosa (tercer muestreo) fueron: Dolichospermun solitarium, Gleocapsa spp., Aphanocapsa delicatissima, Aphanocapsa elachista y Oscillatoria limosa y al inicio de la época seca (cuarto muestreo) las especies dominantes fueron: Microcystis aeruginosa, Gleocapsa spp., Aphanocapsa delicatissima, Oscillatoria limosa y Aulacoseira granulata, por lo que no hubo diferencia muy marcada en la dominancia de especies espacial y temporalmente.

En el cálculo de los índices de similitud se encontró que espacialmente, la mayor similitud se encontró entre Puntilla y el Cacao (E2) y el Punto índice o Aquafinca Saint Peter (E3) y la menor similitud fue entre la desembocadura del Río Varsovia (E1), y la Quebrada Raíces o desembocadura de la quebrada Cianuro (E4). En cambio, la similitud temporal fue mayor entre la época seca en el quinto muestreo (M5) y en el sexto muestreo (M6); la menor entre el muestreo durante la Canícula (M2) y el muestreo durante la época seca del 2015 (M5).

En relación a la riqueza específica, espacialmente la estación localizada frente al Hotel Brisas del Lago (E6) presentó la mayor riqueza específica y el menor valor fue en la estación Punto índice, es decir donde están las balsas de Aquafinca (E3). Temporalmente, la mayor riqueza específica de los muestreos fue en Julio del 2014 correspondiente a la Canícula (M2) y la menor correspondiente Marzo en la época seca del 2015 (M5). Los demás resultados de los índices de riqueza específica, espacial y temporal, fueron muy semejantes entre ellos. 
En la mayor parte del análisis de 180 muestras, además del grupo de Cianophytas y de la acumulación de las mismas en forma de nata verdosa, grumos con mucha espuma y grumos de diferentes tonalidades de verde, también fueron frecuentes especies de la división Chlorophyta (Sphaerocystis schroeteri, Staurastrum leptocladum, Pediastrum simplex, Pediastrum duplex y Eudorina elegans), especies de la división Bacillariophyta (Aulacoseira granulata) y de la división Dinophyta (Peridinium sp.), todas especies indicadoras de lagos eutróficos.

Los procesos de eutroficación producen cambios cuantitativos y cualitativos en la comunidad de fitoplancton, aumentando la frecuencia y densidad de floraciones algales. Cuando los ambientes presentan características eutróficas, generalmente predominan las cianobacterias la mayor parte del año. Es posible que el patrón de lluvia en el lago pudo haber arrastrado cantidades considerables de fósforo y nitrógeno y otras sustancias que afectan el pH básico del agua superficial, turbidez y transparencia. Así que, bajo ciertas condiciones, especialmente donde las aguas son ricas en nutrientes como el fósforo y nitrógeno de origen agrícola y de efluentes cloacales, las cianobacterias pueden multiplicarse hasta alcanzar altas densidades. Esto se evidenció con la abundancia promedio de las cianobacterias en el primer muestreo correspondiente a la época seca e inicio de la época lluviosa en Mayo 2014 que coincidió con el final de un evento de floración algal.

Cada estación de muestreo tiene un componente lacustre y un componente ribereño diferente, con una dinámica propia determinada no solo por los procesos de circulación de nutrientes del lago, sino también por los procesos pluviales y de uso y mal uso de los recursos de cada microcuenca de los cuerpos lóticos que desembocan en cada estación de muestreo (excepto la estación 3 o Punto Indice y la estación 6 frente al Hotel Brisas del Lago); en consecuencia, no se encontró relación entre los nutrientes en las diferentes estaciones de muestreo. Es muy posible que la aparición de algunas especies esté determinada por las algas que se desarrollan en cada sistema lótico que aporta sus aguas al lago de Yojoa, pero este sería un tema a abordar en otros estudios relacionados con el fitoplancton y las particularidades del lago de Yojoa (corrientes prevalecientes de las masas de agua con el manejo de las descargas para la Represa Cañaveral, corrientes internas, corriente superficiales, etc.).

La gestión y control de la eutroficación continuará siendo un reto para científicos y gestores durante varios años. La inversión en investigación científica, en educación pública y en la formación de los responsables de tomar decisiones seguramente es la medida más eficaz para reducir paulatinamente esta problemática local y a nivel puntual orientadas a la reducción de las acciones que aumentan la carga de nutrien- 
tes (fósforo y nitrógeno).

Se recomienda establecer un programa de monitoreo para determinar el aporte de materia orgánica y nutrientes de la actividad de cultivo de tilapia en el lago y el establecimiento de medidas para el control del aporte a la eutroficación producida por las jaulas instaladas en el Punto índice por la Empresa Aquafinca Saint Peter Fish.

\section{AGRADECIMIENTOS}

A Esperanza Izaguirre, Ronald Castellón y Jorge Carranza por su participación en los muestreos y trabajo de campo; al Doctor Oscar Parra e Isis Yelena Montes, su estudiante de doctorado en la Universidad de Concepción en Chile, por revisar la clasificación de algunas de las especies de cianobacterias y por sus sugerencias. También gracias a AMUPROLAGO, por el apoyo en la realización en el trabajo de campo.

Se agradece al Departamento Técnico de la ENEE, por haber facilitado la información hidrológica 2014-2015 de la cuenca del lago de Yojoa y a la Dirección de Investigación Científica y Posgrado de la UNAH por el apoyo en la ejecución financiera del proyecto, ya que este trabajo fue financiado mediante una beca sustantiva.

\section{BIBLIOGRAFÍA}

Anderson, J. \& Weber, C. (1966). A Guide to the Common Diatoms at Water Pollution Surveillance Systems Stations. EE.UU.: U.S. Department of the interior Federal Water Pollution Control Administration.

APHA, AWWA \& WPCF. (1975). Standard Methods for the Examination of Water and Wastewater. EE.UU: Library of Congress Catolog.

Berger, W.H. and Parker, F.L. (1970). Diversity of planktonic Foraminifera in deep sea sediments. Science, 168, 1345-1347.

Bicudo, C. \& Bicudo, R. (1970). Algas de aguas continentales brasileras: claves ilustrada para intificacao de generos. Sao Paulo, Brasil: Fundacao Brasileira para o desenvolvimento Do Encino de Ciencias.

CEPIS. (1996). Agua: un patrimonio para preservar. Día Interamericano del Agua. Lima, Perú. 
Conti, A.L.; Rodríguez, M.I. y Angelaccio, C.M. (2005). Ocurrencia de Cyanobacterias y sus tóxinas (microcistinas) en aguas del río de la Plata: evaluación rápida usando el ensayo Elisa. XX Congreso Nacional del Agua y III Simposio de Recursos Marinos del Cono Sur. Mendoza, Argentina.

Cramér, H. (1999). Mathematical Methods of Statistics. Princenton: Univesity Press. D’Angelo, R.; Ruibal, A.; Lerd, D. and Prosperi, C. (1998). Periodicity and toxin production by Cyanobacteria in an Argentinean lake. En: Gokcecus, H. 1998: Water problems in the Mediterranean countries. Vol.1, 2. 1354.

De la Lanza, G.; Hernández, P. y Carvajal, J.L. (2000). Organismos indicadores de la calidad del agua y de la contaminación (bioindicadores). México: Plaza y Váldez. Edmonson, W. (1959). Fresh Water Biology. EE.UU.: Jonh Wiley and Sons.

Ferguson, E. (1968). Dinoflagellates of the Caribbean Sea and Adjacent areas. EE.UU: University of Miami Press.

García, M. \& López- García, J. (1996). Análisis estadístico para datos categóricos. Editorial Síntesis (Síntesis Psicología). Madrid, España.

Geólogos del mundo. (2011). Ampliación del programa para la regeneraciòn mediambiental del lago de Yojoa. Informe final. Segunda etapa 210-2011. Asturias, España.

González de Infante, A. (1988). El plancton de las aguas continentales. Secretaría General de la Organización de los Estados Americanos. Washington, D.C.

González, E.J.; Ortaz, M.; Peña Herrera, C.; Montes, E. y Matos, M.L. (2003). Fitoplancton de cinco embalses de Venezuela con diferentes estados tróficos. Limnetica, 22 (1-2), 15-35.

González, A.; Otero, L. y Cimadevilla, D. (2011). Ampliación del programa para la regeneracion medioambiental del lago de Yojoa. Santa Cruz de Yojoa: AMUPROLAGO.

Griffith, R. (1961). Phytoplanckton of Chesapeake Bay: An lilustrated Guide to the Genera. EE.UU: Chesapeake Biological Laboratory Maryland Department of Research and Education.

Hernández, R.; Morales, M.R.; Ortega Murillo.; Alvarado Villanueva, R.; Sánchez Heredia, J.D. y Medrano Zarco F. (2008). Variación anual del fitoplancton en el lago cráter La Alberca de Tacámbaro, Michoacán, México. Biológicas, 10, 5-17.

Hornstrom, E. (1981). Trophic characterization of lakes by means of qualiotative phytoplankton analysis. Limnologica, 13(2), 249-261.

House, P. (2002). Diagnóstico ambiental del lago de Yojoa. Honduras: The Nature Conservacy, AMUPROLAGO.

Hutichinson, G.E. (1967). A treatice on limnology. II Introduction to lake bioly and limnology plankton. N.Y.: John Willey and Sons. 
Kebede, E. (1996). Phytoplankton salinity in the Ethiopian Rift Valley. Comprenhensive Summanes of Uppsala Dissertation from the faculty of Science and Technology. Uppsala University. Suecia.

Margalef, R. y Whittaker, R.H. (1992). Evolution and measurement of species diversity. Taxon, 21, 213-251.

McAlice, B.J. (1971). Phytoplankton sampling with the Sedgewick Rafter Cell. Limnol. Oceanogr., 16, 19-28.

Novoa, M.D.; Martínez de Fabricius, A.I.; Luque, M.E.; Lombardo, D. (2011). Distribución temporal del fitoplancton de un lago urbano del centro de Argentina (río Cuarto, Córdoba). Biológicas, 13(2), 1-14.

Otero, L. (2011). Temporalidad de parámetros de calidad en el lago de Yojoa, Honduras. Tesis. Facultad de Geología, Universidad de Oviedo.

Parra, O.; Gonzales, M. y Dellarosa, V. (1983). Manual taxonómico del fitoplancton de aguas continentales con especial referencia al fitoplancton de Chile: Chlorophyceae. Parte I: Volvocales, Tetrasporales, Chlorococcales y Ulothricales. Chile: Universidad de Concepción.

Pizzolon, L. (1996). Importancia de las cianobacterias como factor de toxicidad en las aguas continentales. Interciencia, 21(6), 239-245.

Prescott, G. (1964). The Fresh Water Algae. EE.UU.: W.M.C. Browm Company Publishers.

Prosperi, C. (1999). Las algas microscópicas y su efecto sobre el ambiente. Universidad Libre del Ambiente. 1(1): 2-6. Uruguay.

Reynolds, C. S. (2006). Ecology of phytoplankton. Cambridge: Cambridge University Press.

Ridyng, S.O. y Rast, W. (1992). El control de la eutroficación en lagos y pantanos. Madrid: Ediciones Pirámide, UNESCO.

Rivera, C. (2003). Plan de manejo de la cuenca del lago de Yojoa. Honduras: AMUPROLAGO.

Robarts, R.D. y Zohary, T. (1984). Microcystis aeruginosa and underlight attenuation in a hypertrophic lake. Hartbeespoort Dam, South Africa. J. Ecol., 72, 1001-1018. Romero, F. y Mejía, N. (2007). Batimetría del lago de Yojoa. Técnico-Científica Tatascan, 19(2), 12.

Round, F.M. (1981). The Ecology of algae. New York: Cambridge University Press. Salmaso, N. (2000). Factors affecting the seasonality and distribution of cyanobacteria and chlorophytes. A case study from the large lakes south of the Alps, with special reference to Lake Garda. Hydrobiologia, 438, 43-63.

Sant'Anna, Célia Leite, et al. (2012). Atlas de cianobacterias e microalgas de águas continentais brasileiras. Sao Paulo: Instituto de Botánica.

Scagel, R.F.; Bandoni, R.J.; Maze, J.R.; Rouse, G.E.; Schofield W.B. and Stein, J.R. 
(1987). El reino vegetal: los grupos de plantas y sus relaciones evolutivas. España: Ed. Omega.

Southwood, T. (1978). Ecological Methods with particular refrence to the study of insect populations. Great Britain: Halsted Press Book.

Streble, H. \& Krauter, D. (1985). Atlas de los microorganismos de agua dulce: la vida en una gota de agua. España: Omega.

Studer, E. (2007). Evaluación de parámetros físicos, químicos y biológicos. Indicadores del estado trófico del Lago de Yojoa, Honduras. Tesis de máster Ecole Polythechnique Federale de Lausanne, Suiza.

UNESCO. (2009). Cianobacterias Planctónicas del Uruguay. Manual para la identificación y medidas de gestión. Sylvia Bonilla (editora). Documento Tecnico PHI-LAC, No 16. Montevideo, Uruguay.

Vila, I.; Barends, I. y Montesinos, V. (1987). Abundancia y distribución temporal del fitoplancton en el Embalse Rapel, Chile Central. Revista Chilena de Historia Natural, 60, 37-55. 